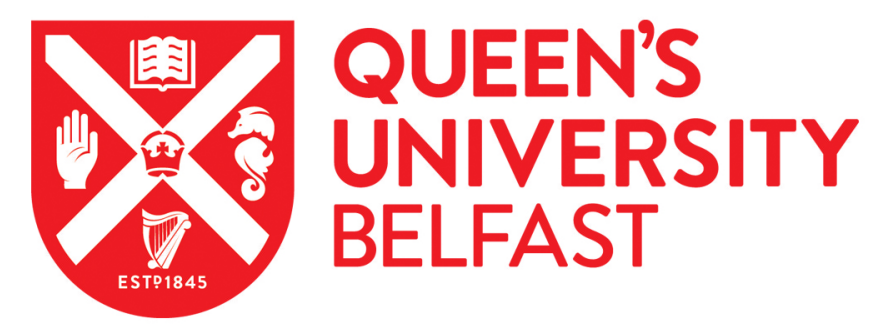

\title{
Theory of electroplasticity based on electromagnetic induction
}

Sutton, A. P., \& Todorov, T. N. (2021). Theory of electroplasticity based on electromagnetic induction. Physical Review Materials, [113605 ]. https://doi.org/10.1103/PhysRevMaterials.5.113605

Published in:

Physical Review Materials

Document Version:

Publisher's PDF, also known as Version of record

Queen's University Belfast - Research Portal:

Link to publication record in Queen's University Belfast Research Portal

Publisher rights

Copyright 2021 American Physical Society.

This work is made available online in accordance with the publisher's policies. Please refer to any applicable terms of use of the publisher.

\section{General rights}

Copyright for the publications made accessible via the Queen's University Belfast Research Portal is retained by the author(s) and / or other copyright owners and it is a condition of accessing these publications that users recognise and abide by the legal requirements associated with these rights.

Take down policy

The Research Portal is Queen's institutional repository that provides access to Queen's research output. Every effort has been made to ensure that content in the Research Portal does not infringe any person's rights, or applicable UK laws. If you discover content in the Research Portal that you believe breaches copyright or violates any law, please contact openaccess@qub.ac.uk. 


\title{
Theory of electroplasticity based on electromagnetic induction
}

\author{
A. P. Sutton $\circledast^{1, *}$ and T. N. Todorov $\circledast^{2, \dagger}$ \\ ${ }^{1}$ Department of Physics, Imperial College London, Exhibition Road, London SW7 2AZ, United Kingdom \\ ${ }^{2}$ Atomistic Simulation Centre, School of Mathematics and Physics, Queen's University Belfast, Belfast BT7 INN, United Kingdom
}

(Received 19 May 2021; accepted 25 October 2021; published 18 November 2021)

\begin{abstract}
In the 1960s, it was discovered in the former Soviet Union that short pulses of intense electric currents through metallic wires brought about temporary drops in the flow stress of the metal as it is deformed plastically. This became known as the electroplastic effect, or electroplasticity. After more than 50 years of experimental and theoretical research, no consensus has emerged as to the mechanism of the effect. Following a brief review of the principal experimental results, we show that when a current flows through a metal the ionic cores of atoms of the metal experience a force equal to the Lorentz force on the conduction electrons, which arises from the magnetic field created by the current. This is the origin of the pinch effect in metals. We then present a new theory of electroplasticity based on mechanical stresses created by pulsing the current as a result of electromagnetic induction. Unlike earlier theories, the rate of change of the current is treated explicitly in a dynamic version of the pinch effect. Pulses of normal and shear stresses arise with a magnitude that depends on the rate at which the current changes during a pulse and a small number of other well defined variables. Unlike earlier theories which focused on the maximum of the current during a pulse, this new theory highlights the time dependence of the current pulse as well as its maximum value. Experiments to test the theory are suggested. Four mechanisms proposed earlier for the electroplastic effect are reviewed critically in the appendices. They are dislocation unpinning in a magnetic field, electromigration of dislocations, Joule heating, and the static pinch effect. We show that the physics of the first two mechanisms is unsound and the second two cannot explain most experimental observations.
\end{abstract}

DOI: 10.1103/PhysRevMaterials.5.113605

\section{INTRODUCTION}

When a pulse of high density electric current is passed through a metallic wire, while it is stretched plastically at a constant rate, it has been experimentally observed that the stress in the wire decreases temporarily. This phenomenon has become known as the electroplastic effect or electroplasticity. It was discovered by Troitiskii in the former Soviet Union in the 1960s [1]. It has been observed in a variety of metals and alloys. The effect has been reproduced in the West by many groups, most notably Conrad and coworkers who have also published excellent reviews [2-4]. Most recently Dimitrov et al. [5] have provided an excellent review of the experimental and theoretical literature on electroplasticity. The majority of this work has considered large current densities, typically between $10^{8}$ and $10^{10} \mathrm{~A} \mathrm{~m}^{-2}$, in wires of diameter $\approx 1 \mathrm{~mm}$. The duration of most commonly used current pulses is between 10 and $100 \mu \mathrm{s}$. Using pulses of much smaller current densities $\left(5 \times 10^{6} \mathrm{~A} \mathrm{~m}^{-2}\right)$ and longer duration $(0.1 \mathrm{~s})$ on titanium 7 at.\% aluminum samples Zhao et al. [6] showed the pulses alter dislocation microstructures and ductility of the alloy. These changes were not found when the current was continuous. Zhao et al. concluded the pulses were essential for the effect. In another study Shibkov et al. [7] showed that pulses of increasing current density $\left(10-50 \times 10^{6} \mathrm{~A} \mathrm{~m}^{-2}\right)$

\footnotetext{
*a.sutton@imperial.ac.uk

†t.todorov@qub.ac.uk
}

applied to certain aluminum - magnesium alloys delayed the onset of jerky flow to higher plastic strains and eventually suppressed it completely. Although these studies on alloys are very interesting the presence of solute atoms complicates the interpretation of the results significantly. For this reason, we shall confine our attention to pure metals.

Electroplasticity has started to be exploited in engineering applications, particularly as a means of facilitating bulk deformation and sheet-metal forming [8,9]. Some of these applications use continuous currents where Joule heating could be significant. There are some instances of pulsed currents being used, which have the advantage of reduced energy costs.

All symbols used in this paper are listed in the table in Appendix A. Several mechanisms have been proposed for electroplasticity in pure metals. The majority of them assume the current is pulsed to reduce Joule heating, but the pulsing has no other purpose or effect. Consequently these explanations apply equally well to continuous currents, although Joule heating may then dominate over other mechanisms. Two prominent explanations center on the interaction between the current and dislocations, and they are reviewed critically in Appendixes B and C. A third explanation is based on the pinch effect, where the wire is contracted by a radial stress due to the current. Again, this has normally been considered by assuming a constant current, as reviewed in Appendix E. We refer to this version of the pinch effect as the static pinch effect because the current does not change in time. The mechanism based on Joule heating by the current is briefly reviewed in Appendix D. 
In contrast to earlier work, we consider the influence of pulsing the current on the generation of transient stresses in pure metals as a result of electromagnetic induction (Sec. V). These stresses arise from a dynamic version of the pinch effect where the time dependence of the current pulse plays a central role. The time-dependent current generates time-dependent Lorentz forces, as described in Secs. V A and V C, which give rise to time-dependent stresses described in Sec. VD. If the width of the conductor is less than the skin depth the induced stresses are described adequately by the static pinch effect. However, when the skin depth is less than the width of the conductor we find larger stresses are created than those predicted by the static pinch effect. Furthermore, these stresses increase extremely rapidly with increasing sample width at constant current density. Since the skin depth is inversely proportional to the square root of the magnetic permeability we find the induced stresses are much larger in body centered cubic iron than in nonmagnetic metals of the same dimensions subjected to the same current pulses. This explains a striking experimental observation about iron that appears in Ref. [10].

Current-induced stresses arise from body forces acting on the crystal lattice. The origin of the body forces is the magnetic Lorentz force acting on the electrons. A central result of this paper is to show in Sec. IV B that these Lorentz forces are the resultant forces transferred from current-carrying electrons to the crystal lattice. We do not consider the response of any defects to the transient stresses, and therefore we are not addressing electroplasticity directly. Instead, we identify the conditions where these stresses are of sufficient magnitude to play a dominant role in accounting for the response of dislocations to current pulses. Since dislocation glide and mechanical twinning require shear stresses we have calculated the von Mises shear stress invariant for the current-induced stresses. The influence of the stresses generated by current pulses is separate from and in addition to the current-induced forces responsible for the electromigration of point defects. It is well known that electromigration brings about mass transport in metals by biasing the diffusional hops of point defects. Gradients in the elastic strains associated with the current-induced stresses give rise to additional forces on point defects and another mechanism of mass transport, separate from electromigration.

\section{OVERVIEW OF EXPERIMENTAL OBSERVATIONS}

Although the literature on electroplasticity is extensive there are relatively few systematic explorations of the parameters affecting it, such as the current density, the shape and duration of the current pulses and their frequency, the width of the sample in relation to the skin depth, the influence of the magnetic permeability of the sample, the orientation of the crystal axes in single crystal samples to the wire axis, the level of interstitial and substitutional point defects, grain size and the degree of prior work hardening. There have also been only a few observations by transmission electron microscopy of dislocation configurations in samples before and after electropulsing treatments. In our view the paucity of systematic experimentation is one of the principal reasons why there is still no widely accepted understanding of the mechanism(s) of electroplasticity. Since the physics of plasticity

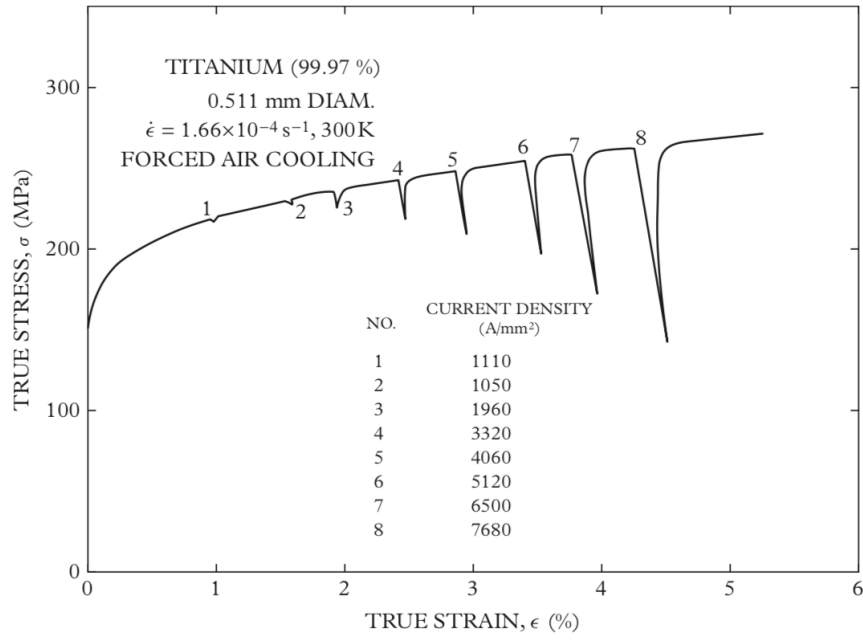

FIG. 1. True stress-true strain relation for a titanium wire deformed in tension at a constant strain rate and subjected to current pulses of increasing current density. Adapted from Okazaki et al. [10]. Copyright (C1978, with permission from Elsevier.

spans length-scales from the atomic (individual dislocations) to the mesoscopic (slip bands) and the macroscopic there is a need for experimental observations of electroplasticity across this range of scales [11].

Another unfortunate aspect of the literature of electroplasticity is that a good deal of the more fundamental experimental and theoretical research has been published in Russian in journals that are not taken by most institutions in the West either in print or electronically.

In a typical experiment, a current is pulsed through a metallic wire as it is stretched at a constant rate and the load is measured as a function of time. The sample is sometimes cooled by immersion in liquid nitrogen or by forced air cooling to minimize the effect of Joule heating. At the beginning of the pulse, the load drops rapidly by up to $40 \%$ of the flow stress, and it returns to its original value more gradually after the pulse. An example is shown in Fig. 1 from the work of Okazaki et al. [10]. It shows the effect of current pulses of $100 \mu$ s duration on the true stress true strain curve of a wire of titanium, $0.51 \mathrm{~mm}$ diameter. The flow stress decreases sharply when the current pulse is applied, and returns over a period of order $0.1 \mathrm{~s}$ to the original stress-strain curve following termination of the pulse. It is noteworthy that the current pulses do not affect the upper envelope of the stress-strain curve.

Okazaki et al. [12] found current pulses produced stress drops also in the elastic part of the stress-strain curve in polycrystalline titanium ( $99.97 \%$ purity). They attributed these stress drops to thermal expansion caused by Joule heating. For a given current density the thermal strain is independent of the dimensions of the wire. This is inconsistent with the observations of Okazaki et al. [12] which showed a quadratic dependence on the radius of the wire at constant current density. However, it is consistent with the pinch effect as the origin of the stress drops in the elastic part of the stress-strain curve. The magnitude of the stress drops observed in the elastic regime are larger by an order of magnitude than those predicted by the static pinch effect. The stress drops in the elastic part of the stress-strain curve were less than those that 
occurred in the plastic part. The extra drop observed in the plastic part of the stress-strain curve has been attributed $[2,13]$ to some form of current-enabled or current-enhanced motion of dislocations. Okazaki et al. [12] found the stress drops in the plastic part of the stress-strain curve increased with the current density. In a further experimental study Okazaki et al. [14] considered the influence of the interstitial content and strain rate on the stress drops in titanium wires. For strain rates between $0.67 \times 10^{-4}$ and $16.7 \times 10^{-4} \mathrm{~s}^{-1}$ they found the strain rate had no influence on the stress drops observed in the plastic part of the stress-strain curve. At a given current density and strain rate, they found the magnitude of the stress drops in both the elastic and plastic parts of the stress-strain curve increased with the interstitial content of the sample.

Troitskii [1] found the load drops in single crystals of zinc were dependent on the orientation of the $c$ axis relative to the wire axis, that they increased with the current density, and they decreased with increasing strain rate. They were observed in compression as well as tension. Load drops have been observed in polycrystalline samples as well as single crystals, and in a variety of metals including $\mathrm{Zn}, \mathrm{Ti}, \mathrm{Nb}, \mathrm{Al}$, $\mathrm{Cu}, \mathrm{Ni}, \mathrm{Fe}, \mathrm{W}, \mathrm{Sn}, \mathrm{Pb}, \mathrm{Cd}$, and some metallic alloys $[2,3,15]$. Troitskii and Spitsyn [16] reported experiments demonstrating that current-enhanced creep rates of single crystals of $\mathrm{Pb}, \mathrm{Zn}$, and $\mathrm{Cd}$ single crystals depended on the direction of the current through the samples. This "polarity effect" is incompatible with an explanation of the effect of the current based either on Joule heating or the pinch effect. Experimental and theoretical research by Troitskii and other Russian authors was reviewed in English by Kir'yanchev et al. [15].

It is sometimes claimed that the observed stress drops vary linearly or quadratically with the current density. We have not found convincing experimental evidence for either. A threshold current density, below which no stress drop is observed, is also often reported. For example, in Fig. 1, such a threshold appears to exists at about $1000 \mathrm{~A} \mathrm{~mm}^{-2}\left(10^{9} \mathrm{~A} \mathrm{~m}^{-2}\right)$. However, the appearance of such a threshold may reflect the insensitivity of the equipment to detect smaller stress drops, such as the much smaller stress drops observed in Appendix D. Troitskii [1] observed that when the stretching of a zinc wire is arrested, so that the tensile stress within it relaxes exponentially to a new finite value, the current pulses continue to create stress drops but they are much smaller. A possibly related observation was made by Okazaki et al. [12] where they saw smaller stress drops in the elastic part of the stressstrain relation than those that appear after yielding.

\section{REVIEW OF PROPOSED MECHANISMS}

Four mechanisms proposed for the electroplastic effect are critically reviewed in Appendices B-E. Pulsing of the current plays no role in any of them and they would apply equally well if the current were constant.

Appendix B discusses a mechanism in which dislocations are unpinned by the magnetic field of the current [13,17-19]. This mechanism is based on the assumption that localized "dangling bond" states exist in metals, which we argue is invalid.

Appendix B discusses electromigration of dislocations, which is the proposal that the current enhances the motion of dislocations by creating additional glide forces on them. For a current-induced force to enhance the glide of a dislocation it has to be dipolar in character to create a resolved shear stress on the dislocation. That is because, in contrast to point defects, the glide force on a dislocation is unaffected by a monopole body force. Using symmetry arguments we show that such current-induced forces are independent of the sign of either the Burgers vector or the line direction. Consequently dislocation loops would not expand under the influence of such forces, and there would be no change in the plastic strain. We conclude that even if such current-induced glide forces exist they cannot account for the observed stress drops.

Appendix D discusses briefly the suggestion that the stress drops are caused by thermal expansion. There are some experiments where Joule heating could play a role, and others where the pulse duration is so short the heating is insignificant. There are experiments carried out under compressive loading where Joule heating would increase the observed stress, whereas it is observed that the current pulse decreases the stress [1].

Finally, in Appendix E, we review the static pinch effect. The use of the adjective "static" is to convey the absence of any time dependence in the current. In Sec. V, the "dynamic" pinch effect is considered, where the time dependence of the current is treated explicitly. We find that the stress drops produced by the static pinch effect are too small to account for those observed experimentally.

In conclusion none of these four mechanisms offers a convincing explanation of the experimentally observed stress drops due to current pulses. We noted at the beginning of this section that none of them depends on the pulsed nature of the current. In contrast, the mechanism we propose in Sec. V depends explicitly on the pulsing of the current. In the next section, we discuss the forces on atoms in metals arising from electric currents, where we will see that the magnetic Lorentz force has a unique significance. This was implicit in earlier treatments, and in our derivation in Appendix E, of the static pinch effect. In the next section, it is justified using the zero sum rule of electromigration [20]. This puts the theory in $\mathrm{Sec}$. V on a firm foundation.

\section{FORCES ON ATOMS IN A METAL DUE TO ELECTRIC CURRENTS}

\section{A. Electric fields in metals and the zero sum rule}

Throughout Sec. IV the frame of reference is the rest frame of the wire, which is also the laboratory frame. Consider a homogeneous, cylindrical, crystalline, metallic wire carrying an electric current. The metal comprises conduction electrons and ions. Each ion consists of the atomic nucleus and core electrons which remain bound to the nucleus. Conduction electrons drift in response to the applied electric field according to Ohm's law. Their drift speed $v_{e}$ is much less than the speed of light $c$. The charge relaxation time of the electrons $\tau_{e}$ is equal to $\epsilon_{0} / \sigma_{c}$, where $\epsilon_{0}$ is the permittivity of free space and $\sigma_{c}$ is the electric conductivity. This is approximately the time taken by conduction electrons in a metal to screen a charge. For most metals it is of order $10^{-17}-10^{-18} \mathrm{~s}$. Since the Thomas-Fermi screening length in a metal is comparable to the interatomic distance and the charge relaxation time is so small, local charge neutrality is maintained at all times except 
at plasmon frequencies when electrons and ions are induced to move in opposite directions.

Since local charge neutrality is maintained at all times, even when the current is pulsed, the total momentum of electrons entering and leaving each transverse slice of the wire per unit time does not change. This remains true even when there are defects present. It follows that there is no net force on the conduction electrons. There are two contributions to the net force on conduction electrons: the force exerted by the applied electric field and the force exerted by the ions. The force exerted by conduction electrons on ions is equal and opposite to the force exerted by ions on conduction electrons. The force exerted by the applied electric field on ions is equal and opposite to the force it exerts on conduction electrons. Therefore the net force on ions is equal and opposite to the net force on conduction electrons. Since the latter is zero there is no net force on ions due to an electric field. This is the zero sum rule introduced in [20]. It follows that there is no net force on a metallic conductor due to an electric field even if it induces a current to flow. This statement assumes there are no other forces acting on the conductor, such as may be applied through its attachment to a substrate. In a free-standing metallic conductor, electromigration of atoms during the passage of a current does not result in a displacement of its center of mass. However, if the conductor is attached to a substrate, there is a net force acting on the conductor and its center of mass is displaced during electromigration.

\section{B. Forces of a magnetic origin}

The zero sum rule is modified when there are magnetic fields present inside a metallic conductor. Electrons driven along a wire by an applied electric field create a magnetic field inside (and outside) the conductor. As a result there is a Lorentz force on the conduction electrons. The Lorentz force on the electrons is directed towards the axis of the wire. To maintain local charge neutrality it is balanced by a force $-\rho_{0} \mathcal{E}(r)$ due to a radial electric field $\mathcal{E}(r)$, sometimes called a Hall field. Here, $-\rho_{0}=-n e$ is the conduction electron charge density, where $n$ is the number of conduction electrons per unit volume and $e$ is the electronic charge. The ionic charge density is $\rho_{0}$. Conduction electrons also experience a force exerted by ions, as in the previous section. The net force on the electrons remains zero because in any transverse slice the momentum of electrons entering and leaving the slice per unit time is the same, provided local charge neutrality is maintained.

Let $\mathbf{F}_{e}(\mathbf{r})$ be the net force per unit volume acting on conduction electrons at $\mathbf{r}$. Let $\mathbf{F}_{e}^{i}(\mathbf{r})$ be the electrostatic force per unit volume ions exert on conduction electrons at $\mathbf{r}$. Let $\mathbf{F}_{e}^{E}(\mathbf{r})$ be the force per unit volume the applied and Hall electric fields exert on conduction electrons at $\mathbf{r}$. Let $\mathbf{F}_{e}^{B}(\mathbf{r})$ be the force per unit volume the magnetic field exerts on conduction electrons at $\mathbf{r}$. Then

$$
\mathbf{F}_{e}(\mathbf{r})=\mathbf{F}_{e}^{i}(\mathbf{r})+\mathbf{F}_{e}^{E}(\mathbf{r})+\mathbf{F}_{e}^{B}(\mathbf{r})=\mathbf{0} .
$$

The net force on the ions arises from the electron-ion electrostatic interaction and the force due the applied and Hall electric fields. These forces are $-\mathbf{F}_{e}^{i}(\mathbf{r})$ and $-\mathbf{F}_{e}^{E}(\mathbf{r})$, respectively. We see from Eq. 1 that their sum is the Lorentz force on the electrons $\mathbf{F}_{e}^{B}(\mathbf{r})$. Therefore the net force on the ions due to the current is identical to the Lorentz force acting on the conduction electrons. This is a counter-intuitive result because the ions are not moving, and therefore the magnetic field does not exert a Lorentz force on them directly. Instead, the Lorentz force is transferred to the ions by the conduction electrons through their electrostatic interaction. Forces acting on conduction electrons of a magnetic origin have a particular significance for electroplasticity because they create net body forces on ions which are the sources of stresses throughout the conductor.

\section{THE DYNAMIC PINCH EFFECT}

\section{A. Electromagnetic induction in a cylindrical wire}

In this Sec. we encounter for the first time physics that arises only when the current is pulsed. The time dependence of a current pulse introduces a time dependence into the associated magnetic field. Faraday's law of induction tells us that an electric field is induced in the wire by the time-dependent magnetic field. In an infinitely long wire, the induced electric field is parallel to the wire. It alters the drift velocity of electrons. It also affects the radial distribution of the current density. The changes in the drift velocity and radial distribution of the current density alter the magnetic field in the wire. All these changes affect the Lorentz forces acting on the electrons, and hence the resultant forces acting on the ions and the stresses they generate.

Following the treatment of the static pinch effect in Appendix E, we consider an infinitely long, homogeneous, cylindrical wire, and we use the same cylindrical coordinate system. The translational invariance of the wire ensures that the electric and magnetic fields and the current density do not vary with position along the wire axis $z$. The azimuthal invariance of the wire ensures their independence of the angle $\phi$. The independent variables are therefore the radial distance $r$ from the axis of the wire and time $t$. The drift velocity $\mathbf{v}_{e}(r, t)$ and the current density $\mathbf{j}(r, t)$ are directed along the wire axis.

The timescale in which the current density, electric fields and magnetic field change in any practical current pulse is many orders of magnitude greater than the charge relaxation time $\tau_{e}$. The electronic charge distribution within the conductor adjusts virtually instantaneously to changes in the Lorentz force. Consequently, the Hall field cancels the Lorentz force at all times. It follows that the only forces on electrons are due to the electric fields parallel to the wire axis. They are the time-dependent, externally applied electric field $\mathbf{E}_{\text {ext }}(t)$ and the induced electric field $\mathbf{E}_{i}(\mathbf{r}, t)$. According to Ohm's law, we have

$$
\mathbf{j}(r, t)=\sigma_{c}\left[\mathbf{E}_{\text {ext }}(t)+\mathbf{E}_{i}(r, t)\right] .
$$

The time-dependent magnetic field induces the electric field $\mathbf{E}_{i}(\mathbf{r}, t)$ along the wire axis through Faraday's law of electromagnetic induction. It is expressed as usual by the following Maxwell equation:

$$
\nabla \times \mathbf{E}_{i}(\mathbf{r}, t)=-\frac{\partial \mathbf{B}(\mathbf{r}, t)}{\partial t} .
$$

Introducing the vector potential $\mathbf{A}(\mathbf{r}, t)$, where $\mathbf{B}(\mathbf{r}, t)=\nabla \times$ $\mathbf{A}(\mathbf{r}, t)$, the induced electric field is $\mathbf{E}_{i}(\mathbf{r}, t)=-\partial \mathbf{A}(\mathbf{r}, t) / \partial t$. The vector potential is also directed along the wire axis. The divergence of the vector potential is zero automatically owing to the translational invariance along the wire axis. 
We also need the following Maxwell equation:

$$
\nabla \times \mathbf{B}(\mathbf{r}, t)=\mu \mathbf{j}(\mathbf{r}, t)+\mu \tau_{e} \frac{\partial \mathbf{j}(\mathbf{r}, \mathbf{t})}{\partial t} .
$$

The magnetic permeability $\mu$ is equal to $\mu_{r} \mu_{0}$, where $\mu_{0}$ is the permeability of free space and $\mu_{r}$ is the relative permeability of the wire. The second term on the right originates from the displacement current. It is negligible in comparison to the first term, again because $\tau_{e}$ is so small. Therefore this Maxwell equation reduces to Ampère's law:

$$
\nabla \times \mathbf{B}(\mathbf{r}, t)=\mu \mathbf{j}(\mathbf{r}, t) .
$$

It follows from Eqs. (2), (3), (5), and $\nabla \cdot \mathbf{E}_{i}=0$ that

$$
\frac{1}{\sigma_{c} \mu} \nabla^{2} \mathbf{j}(\mathbf{r}, t)=\frac{\partial \mathbf{j}(\mathbf{r}, t)}{\partial t} \text {. }
$$

Similar vector diffusion equations may be derived for $\mathbf{E}(\mathbf{r}, t)$, $\mathbf{B}(\mathbf{r}, t)$, and $\mathbf{A}(\mathbf{r}, t)$. These equations are well known in the context of the skin effect in AC conductors, for example see Smythe [21]. Since $\mathbf{j}$ is directed along the wire axis Eq. (6) simplifies as follows:

$$
\frac{\partial^{2} j(r, t)}{\partial r^{2}}+\frac{1}{r} \frac{\partial j(r, t)}{\partial r}-\sigma_{c} \mu \frac{\partial j(r, t)}{\partial t}=0 .
$$

Once Eq. (7) is solved the magnitude of the magnetic field may be calculated as follows:

$$
B(r, t)=\frac{\mu}{r} \int_{0}^{r} d r^{\prime} r^{\prime} j\left(r^{\prime}, t\right) .
$$

The Lorentz force on the conduction electrons is then given by

$$
\mathbf{f}_{L}(r, t)=-j(r, t) B(r, t) \widehat{\mathbf{r}} .
$$

This is a body force that acts on the ions during a current pulse. Note that for a given current density distribution the Lorentz force is proportional to the magnetic permeability. This indicates that wires with higher magnetic permeabilities may have larger pulse-induced body forces. When the Lorentz body force is substituted into the equation of mechanical equilibrium, Eq. (E3), it yields the stress field due to the current pulse. Note also that the time dependence of the stress field does not have to be treated elastodynamically provided $c_{l} t_{p} \gg r_{m}$, where $c_{l}$ is the longitudinal speed of sound in the wire and $t_{p}$ is the pulse duration, which is always true in the experiments of which we are aware.

\section{B. General solution of the equations for a cylindrical wire}

The vectors $\mathbf{j}, \mathbf{E}, \mathbf{E}_{\text {ext }}$, and $\mathbf{E}_{i}$ are directed along the wire axis. The magnetic field $\mathbf{B}$ is directed along the azimuthal direction. They are all functions of $r$ and $t$, except $\mathbf{E}_{\text {ext }}$ which is a function of $t$ only. Equation (3) simplifies to

$$
\frac{\partial E(r, t)}{\partial r}=\frac{\partial B(r, t)}{\partial t}
$$

and Eq. (5) simplifies to

$$
\frac{1}{r} \frac{\partial(r B(r, t))}{\partial r}=\mu j(r, t),
$$

which is the differential form of Eq. (8).

The externally applied electric field $E_{\text {ext }}(t)$ of the pulse drives the current $j(r, t)$. The task of this Sec. is to find the relationship between $j(r, t)$ and $E_{\text {ext }}(t)$. Along the way we shall also derive expressions for $B(r, t)$ and $E_{i}(r, t)$. Introducing the Fourier transform in the time variable, we have

$$
\begin{gathered}
\bar{j}(r, \omega)=\int_{-\infty}^{\infty} j(r, t) e^{-i \omega t} d t, \\
j(r, t)=\frac{1}{2 \pi} \int_{-\infty}^{\infty} \bar{j}(r, \omega) e^{i \omega t} d \omega .
\end{gathered}
$$

The transforms $\bar{B}(r, \omega)$ and $\bar{E}_{i}(r, \omega)$ are similarly defined. The transform $\bar{E}_{\text {ext }}(\omega)$ is slightly different because it has no positional dependence:

$$
\begin{aligned}
& \bar{E}_{\mathrm{ext}}(\omega)=\int_{-\infty}^{\infty} E_{\mathrm{ext}}(t) e^{-i \omega t} d t, \\
& E_{\mathrm{ext}}(t)=\frac{1}{2 \pi} \int_{-\infty}^{\infty} \bar{E}(\omega) e^{i \omega t} d \omega .
\end{aligned}
$$

When Eq. (7) is transformed it becomes

$$
\frac{\partial^{2} \bar{j}}{\partial r^{2}}+\frac{1}{r} \frac{\partial \bar{j}}{\partial r}-\frac{i}{\lambda_{\omega}^{2}} \bar{j}=0,
$$

where $\lambda_{\omega}=1 / \sqrt{\mu \sigma_{c} \omega}$ is a diffusion length associated with the angular frequency $\omega$. The solution of this equation is as follows:

$$
\bar{j}(r, \omega)=\alpha(\omega) J_{0}\left(i^{3 / 2} \frac{r}{\lambda_{\omega}}\right) .
$$

Here, $J_{0}$ is the Bessel function of the first kind of order zero. It has a complex argument. The prefactor $\alpha(\omega)$ is a complex amplitude that is a function of $\omega$ only and it is to be determined by relating it to $\bar{E}_{\text {ext }}(\omega)$. The average current density $\bar{J}(\omega)$ over all radii at this angular frequency is then

$$
\begin{aligned}
\bar{J}(\omega) & =\frac{1}{\pi r_{m}^{2}} \int_{0}^{r_{m}} 2 \pi r \bar{j}(r, \omega) d r \\
& =2 \alpha(\omega)\left(i^{-3 / 2} \frac{\lambda_{\omega}}{r_{m}}\right) J_{1}\left(i^{3 / 2} \frac{r_{m}}{\lambda_{\omega}}\right) .
\end{aligned}
$$

Inserting Eq. (17) into Eq. (8), we obtain an equation for $\bar{B}(r, \omega)$ :

$$
\bar{B}(r, \omega)=i^{-3 / 2} \mu \alpha(\omega) \lambda_{\omega} J_{1}\left(i^{3 / 2} \frac{r}{\lambda_{\omega}}\right),
$$

where $J_{1}$ is the Bessel function of the first kind of order one. Note that in the limit $\omega \rightarrow 0, \bar{B}(r, \omega) \rightarrow \mu \alpha(0) r / 2$, in agreement with Eq. (E1) if we identify $\alpha(0)$ with the constant current density $j_{0}$.

Since $E_{\text {ext }}(t)$ is assumed to have no spatial dependence the electric field in Eq. (10) can be replaced by the induced field $E_{i}$. Integrating this equation, we obtain the following expression for $\bar{E}_{i}(r, \omega)$ :

$$
\bar{E}_{i}(r, \omega)=-\mu \omega \lambda_{\omega}^{2} \alpha(\omega)\left\{1-J_{0}\left(i^{3 / 2} \frac{r}{\lambda_{\omega}}\right)\right\} .
$$

The induced electric field $\overline{\mathcal{E}}_{i}(\omega)$, averaged over the crosssection is as follows:

$$
\begin{aligned}
\overline{\mathcal{E}}_{i}(\omega) & =\frac{1}{\pi r_{m}^{2}} \int_{0}^{r_{m}} 2 \pi r \bar{E}_{i}(r, \omega) d r \\
& =-\alpha(\omega) \mu \lambda_{\omega}^{2} \omega\left[1-2\left(i^{-3 / 2} \frac{\lambda_{\omega}}{r_{m}}\right) J_{1}\left(i^{3 / 2} \frac{r_{m}}{\lambda_{\omega}}\right)\right] .
\end{aligned}
$$

Using Ohm's law, Eq. (2), we have the following relationship between the total current density, Eq. (18), the externally 
applied electric field $\bar{E}_{\text {ext }}(\omega)$ and the induced electric field averaged over all radii, Eq. (21):

$$
\begin{aligned}
\bar{J}(\omega) & =\frac{1}{\pi r_{m}^{2}} \int_{0}^{r_{m}} 2 \pi r j(r, \omega) d r \\
& =\frac{\sigma_{c}}{r_{m}^{2}} \int_{0}^{r_{m}} 2 r\left(\bar{E}_{\mathrm{ext}}(\omega)+\bar{E}_{i}(r, \omega)\right) d r \\
& =\sigma_{c}\left(\bar{E}_{\mathrm{ext}}(\omega)+\overline{\mathcal{E}}_{i}(\omega)\right) .
\end{aligned}
$$

Substituting $\bar{J}(\omega)$ from Eq. (18) and $\overline{\mathcal{E}}_{i}(\omega)$ from Eq. (21) into this equation, we obtain the explicit relationship we sought between $\alpha(\omega)$ and $\bar{E}_{\text {ext }}(\omega)$ :

$$
\alpha(\omega)=\sigma_{c} \bar{E}_{\text {ext }}(\omega)
$$

Before we leave this Sec. we note a general feature of the induced current at each radius, $j_{i}(r, t)=\sigma_{c} E_{i}(r, t)$. The integral of $j_{i}(r, t)$ over all time at each radius $r$ is zero:

$$
\begin{aligned}
\int_{-\infty}^{\infty} j_{i}(r, t) d t & =\sigma_{c} \int_{-\infty}^{\infty} E_{i}(r, t) d t \\
& =\frac{\sigma_{c}}{2 \pi} \int_{-\infty}^{\infty} d t \int_{-\infty}^{\infty} \bar{E}_{i}(r, \omega) e^{i \omega t} d \omega \\
& =\frac{\sigma_{c}}{2 \pi} \int_{-\infty}^{\infty} \bar{E}_{i}(r, \omega) 2 \pi \delta(\omega) d \omega \\
& =\sigma_{c} \bar{E}_{i}(r, 0) \\
& =0 .
\end{aligned}
$$

In the third line, $\delta(\omega)$ is the Dirac delta function. The last step follows from Eq. (20) and $J_{0}(0)=1$. Thus, in each cylindrical shell of radius $r$ and thickness $d r$, the induced current averaged over all time is zero. It follows that at each radius the induced current must change direction with time so that over the duration of the pulse it averages to zero. However, if the current passing through the wire changes from one constant value to a different constant value, then this relation no longer holds and there is a net induced current at each finite radius of the wire. This follows directly from integrating Eq. (10) with respect to $t$ and $r$ :

$$
\begin{aligned}
\int_{-\infty}^{\infty}\left\{E_{i}(r, t)-E_{i}(0, t)\right\} d t & =\int_{-\infty}^{\infty} E_{i}(r, t) d t \\
& =\int_{r^{\prime}=0}^{r}\left\{B\left(r^{\prime}, \infty\right)-B\left(r^{\prime},-\infty\right)\right\} d r^{\prime}
\end{aligned}
$$

The first equality follows because there is no magnetic field at $r=0$ and therefore $E_{i}(0, t)=0$ at all $t$. The right-hand side is zero only if the current flowing in the wire is the same long before and after the change.

\section{Lorentz forces created by a Lorentzian current pulse}

To make further progress, we have to choose a functional form for the pulse $E_{\text {ext }}(t)$. For short pulses where the externally applied electric field does not reach a constant value for a large fraction of the pulse duration the choice of the functional form is largely a matter of mathematical convenience. For longer pulses, it may be more appropriate to choose a rounded rectangular form to capture the relatively rapid rise and fall of $E_{\text {ext }}(t)$.

Since we have short pulses in mind we have chosen a Lorentzian function:

$$
E_{\mathrm{ext}}(t)=\frac{\varepsilon}{\pi} \frac{p}{p^{2}+t^{2}},
$$

where $2 p$ is the full width at half maximum of the pulse and $\varepsilon /(\pi p)$ is its maximum value. Note that the electric field $E_{\text {ext }}(t)$ rises when $t<0$, reaching a maximum at $t=0$, and falls symmetrically when $t>0$. In the limit $p \rightarrow \infty$, the pulse broadens into a constant electric field $E_{\text {ext }}=\varepsilon /(\pi p)$. Thus, if $\varepsilon \rightarrow \infty$ as $p \rightarrow \infty$ such that $\varepsilon / p$ remains finite Eq. (26) describes a constant finite electric field $E_{\text {ext }}=\varepsilon /(\pi p)$. This provides a useful check on the results of this Sec. because in this limit they should reproduce the results of Appendix E. On the other hand, by taking the limit $p \rightarrow 0$ the pulse $E_{\text {ext }}(t)$ becomes $\varepsilon \delta(t)$. However, this limit violates, our assumption that the contribution of the term arising from the displacement current in Eq. (4) can be neglected. Therefore $p$ can be made small but not zero. The Fourier transform of the pulse is $\bar{E}_{\text {ext }}(\omega)=\varepsilon e^{-p|\omega|}$.

Inserting the Fourier transform of the Lorentzian into Eq. (17) and using Eq. (23), we obtain the following expression for the Fourier transform of the current density due to a Lorentzian pulse:

$$
\bar{j}(r, \omega)=\sigma_{c} \varepsilon e^{-p|\omega|} J_{0}\left(i^{3 / 2} r \sqrt{\mu \sigma_{c} \omega}\right) .
$$

The current density $j(r, t)$ is obtained from the inverse Fourier transform by expanding the Bessel function as an infinite series, integrating each term and summing the resulting series:

$$
\begin{aligned}
j(r, t) & =\operatorname{Re}\left[\frac{\sigma_{c} \varepsilon}{\pi} \int_{0}^{\infty} \sum_{k=0}^{\infty} \frac{\left(i \mu \sigma_{c} r^{2} \omega / 4\right)^{k}}{(k !)^{2}} e^{\omega(i t-p)} d \omega\right] \\
& =\operatorname{Re}\left[\frac{\sigma_{c} \varepsilon}{\pi} \frac{1}{p-i t} \sum_{k=0}^{\infty}\left(\frac{-\mu \sigma_{c} r^{2} / 4}{t+i p}\right)^{k} \frac{1}{k !}\right] \\
& =\operatorname{Re}\left[\frac{\sigma_{c} \varepsilon}{\pi} \frac{(p+i t)}{\left(p^{2}+t^{2}\right)} \exp \left\{\frac{-\mu \sigma_{c} r^{2}(t-i p)}{4\left(p^{2}+t^{2}\right)}\right\}\right] \\
& =\frac{\sigma_{c} \varepsilon}{\pi} \frac{\exp \left[-\left(\mu \sigma_{c} r^{2} t / 4\left(p^{2}+t^{2}\right)\right)\right]}{\sqrt{p^{2}+t^{2}}} \cos \left(\frac{\mu \sigma_{c} r^{2} p}{4\left(p^{2}+t^{2}\right)}+\tan ^{-1}(t / p)\right) .
\end{aligned}
$$


TABLE I. Diffusion lengths of nominally pure metals for current pulses with a full width $(2 p)$ at half maximum of $60 \mu$ s. The electrical conductivities $\sigma_{c}$, at $20^{\circ} \mathrm{C}$, are from Ref. [22]. For all the metals except iron the magnetic permeability is $\mu_{0}=4 \pi \times 10^{-7} \mathrm{NA}^{-2}$. For pure iron the relative magnetic permeability is between 1000 and $1.4 \times 10^{6}$ depending on the heat treatment applied to the sample [23].

\begin{tabular}{lcccccc}
\hline \hline & $\mathrm{Al}$ & $\mathrm{Au}$ & $\mathrm{Cu}$ & $\mathrm{Ti}$ & $\mathrm{Zn}$ & $\mathrm{Fe}$ \\
\hline$\sigma_{c}\left(\Omega^{-1} \mathrm{~m}^{-1}\right)$ & $3.77 \times 10^{7}$ & $4.26 \times 10^{7}$ & $5.98 \times 10^{7}$ & $2.38 \times 10^{6}$ & $1.69 \times 10^{7}$ & $1.03 \times 10^{7}$ \\
$L_{D}$ & $1.1 \mathrm{~mm}$ & $1.1 \mathrm{~mm}$ & $0.89 \mathrm{~mm}$ & $4.5 \mathrm{~mm}$ & $1.7 \mathrm{~mm}$ & $1.8-70 \mu \mathrm{m}$ \\
\hline \hline
\end{tabular}

We have verified that Eq. (31) satisfies Eq. (16). It is useful to introduce the following dimensionless variables:

$$
\begin{gathered}
\mathcal{R}=r / L_{D}, \\
\mathcal{T}=t /(2 p) .
\end{gathered}
$$

The parameter $L_{D}$ is a characteristic diffusion length that depends on the electrical conductivity and magnetic permeability of the metal and the duration of the pulse:

$$
L_{D}=\sqrt{2 p /\left(\mu \sigma_{c}\right)} .
$$

$L_{D}$ can be made smaller by decreasing the pulse duration, subject to the limitation stated above. It is the same as the skin depth for an alternating current of angular frequency $1 / p$. In Table I, we have calculated $L_{D}$ in a variety of pure metals assuming $2 p=60 \mu$ s. Notice the exceptionally small range of diffusion lengths in iron, which is a consequence of its range of high magnetic permeabilities.

In terms of the dimensionless variables, $j(\mathcal{R}, \mathcal{T})$ is expressed as follows:

$$
\begin{aligned}
j(\mathcal{R}, \mathcal{T})= & \sigma_{c} \frac{\varepsilon}{\pi p} \frac{\exp \left[-\mathcal{R}^{2} \mathcal{T} /\left(1+4 \mathcal{T}^{2}\right)\right]}{\sqrt{1+4 \mathcal{T}^{2}}} \\
& \times \cos \left(\frac{\mathcal{R}^{2}}{2\left(1+4 \mathcal{T}^{2}\right)}+\tan ^{-1}(2 \mathcal{T})\right) .
\end{aligned}
$$

In the limit $\varepsilon \rightarrow \infty$ and $p \rightarrow \infty$, such that $\varepsilon / p$ remains finite, Eq. (35) correctly becomes the constant current density $j=\sigma_{c}[\varepsilon /(\pi p)]$. If the diffusion length $L_{D}$ is much larger than

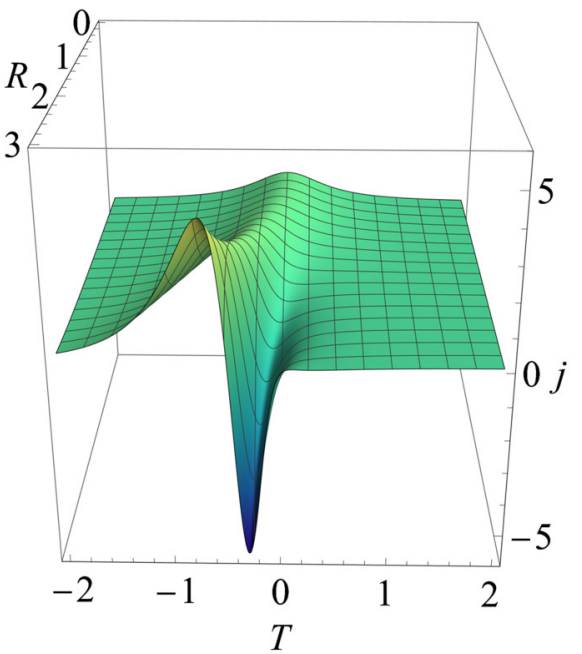

the radius $r_{m}$ of the wire then the current density becomes $\sigma_{c} E_{\text {ext }}(t)$, independent of position within the wire. This may be described as the quasistatic limit because the time dependence of the current density is the same as that of the external electric field. When $L_{D}<r_{m}$ the current density can display a dependence on position and time which differs markedly from that of the external electric field. Perhaps the most striking feature in this limit is the oscillatory dependence of the current density on position and time, resulting from electromagnetic induction. These features are displayed in the plot of $j(\mathcal{R}, \mathcal{T})$ in the left panel of Fig. 2. The plot shows a rapid increase in the peak current density at $\mathcal{R}>1$, and at $\mathcal{R}>2$ the rise is followed by an abrupt reversal of the current before the peak is reached in the electric field at $\mathcal{T}=0$. The current flowing in the wire at $\mathcal{T}>0$ decreases rapidly at $\mathcal{R}>2$ even though at $\mathcal{T}=0$ the applied electric field is a maximum. The symmetry of the applied electric field on either side of $\mathcal{T}=0$ is broken in the current density except when $\mathcal{R} \ll 1$. The panel on the right of Fig. 2 shows the induced current density $j_{i}(\mathcal{R}, \mathcal{T})$. According to Eq. (24) the induced current density at each radius averaged over all time is zero.

The magnetic field $B(r, t)$ may be found by a similar set of steps using Eqs. (19), (23) and the Fourier transform $\bar{E}_{\text {ext }}(\omega)=\varepsilon e^{-p|\omega|}$. The result is as follows:

$$
B(r, t)=\frac{2 \varepsilon}{\pi r} \exp \left[-\left(\mu \sigma_{c} r^{2} t / 4\left(p^{2}+t^{2}\right)\right)\right] \sin \left(\frac{\mu \sigma_{c} r^{2} p}{4\left(p^{2}+t^{2}\right)}\right)
$$

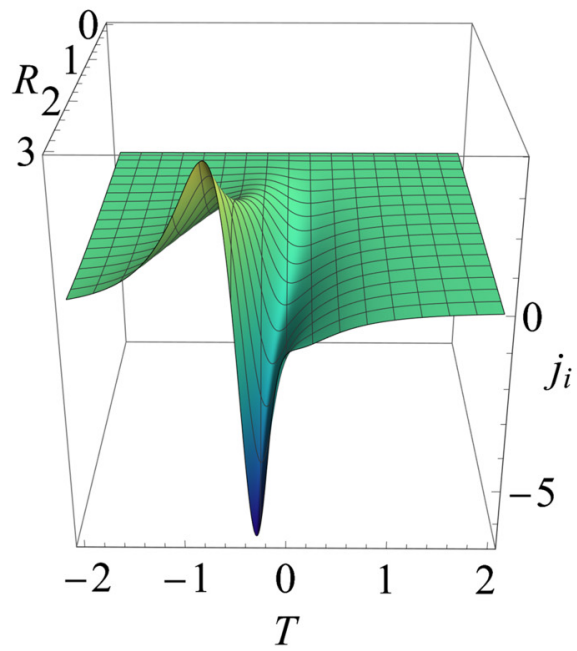

FIG. 2. (Left) Plot of the total current density $j(\mathcal{R}, \mathcal{T})$ according to Eq. (35). (Right) Plot of the induced current density $j_{i}(\mathcal{R}, \mathcal{T})$, which is the difference $j(\mathcal{R}, \mathcal{T})-\sigma_{c} E_{\text {ext }}(\mathcal{T})$. The current densities are in units of $\sigma_{c} \varepsilon /(\pi p)$. 


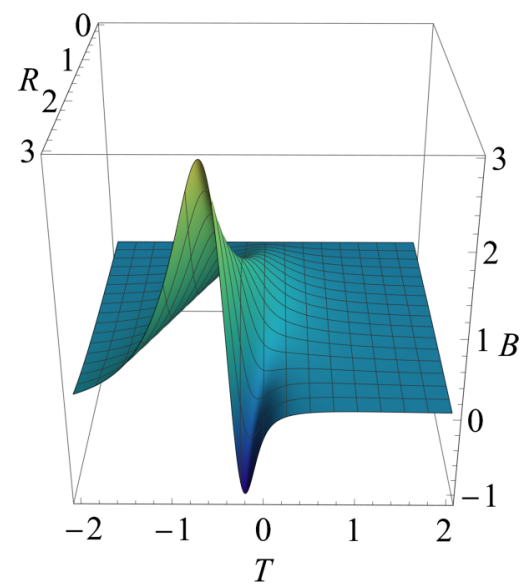

FIG. 3. Plot of the magnetic field $B(\mathcal{R}, \mathcal{T})$ according to Eq. (37). The magnetic field $B$ is in units of $2 \varepsilon /\left(\pi L_{D}\right)$.

$$
\begin{aligned}
B(\mathcal{R}, \mathcal{T})= & \frac{\varepsilon}{\pi p} \frac{2 p}{L_{D}} \frac{\exp \left[-\mathcal{R}^{2} \mathcal{T} /\left(1+4 \mathcal{T}^{2}\right)\right]}{\mathcal{R}} \\
& \times \sin \left(\frac{\mathcal{R}^{2}}{2\left(1+4 \mathcal{T}^{2}\right)}\right) .
\end{aligned}
$$

In the limit $\varepsilon \rightarrow \infty$ and $p \rightarrow \infty$ such that $\varepsilon / p$ remains finite, Eq. (36) becomes the time-independent magnetic field $B(r)=$

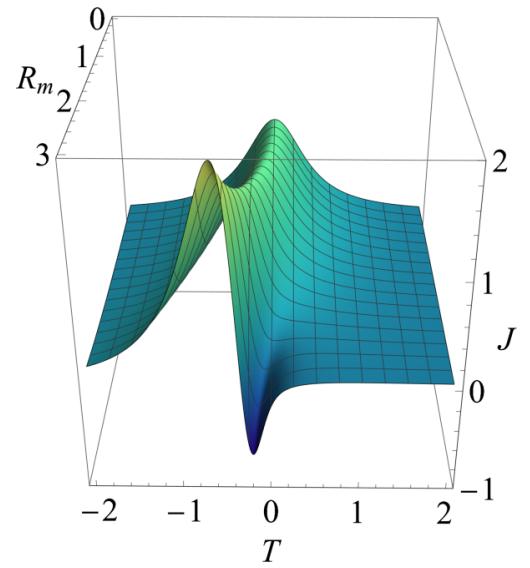

FIG. 4. Plot of the total current density $J$ as a function of $\mathcal{R}_{m}$ and $\mathcal{T}$ according to Eq. (40). The total current density is in units of $\sigma_{c}(\varepsilon /(\pi p))$. Note the increasing departure with increasing $\mathcal{R}_{m}$ of the total current density from the Lorentzian form, $\sigma_{c} E_{\text {ext }}(t)$, seen at $\mathcal{R}_{m}=0$.

$\mu j r / 2$, where $j=\sigma_{c} \varepsilon /(\pi p)$, in agreement with Eq. (E1). Figure 3 shows a plot of the magnetic field $B(\mathcal{R}, \mathcal{T})$.

The total current density $J(t)$ is related to the magnetic field at $r=r_{m}$ through Ampère's law:

$$
\begin{aligned}
J(t) & =\frac{1}{\pi r_{m}^{2}} \int_{0}^{r_{m}} 2 \pi r j(r, t) d r=\frac{2}{r_{m}} \frac{B\left(r_{m}, t\right)}{\mu} \\
& =2 \sigma_{c} \frac{\varepsilon}{\pi p} \frac{2 p}{\mu \sigma_{c} r_{m}^{2}} \exp \left[-\left(\mu \sigma_{c} r_{m}^{2} t / 4\left(p^{2}+t^{2}\right)\right)\right] \sin \left(\frac{\mu \sigma_{c} r_{m}^{2} p}{4\left(p^{2}+t^{2}\right)}\right), \\
J(\mathcal{T}) & =2 \sigma_{c} \frac{\varepsilon}{\pi p} \frac{\exp \left[-\mathcal{R}_{m}^{2} \mathcal{T} /\left(1+4 \mathcal{T}^{2}\right)\right]}{\mathcal{R}_{m}^{2}} \sin \left(\frac{\mathcal{R}_{m}^{2}}{2\left(1+4 \mathcal{T}^{2}\right)}\right),
\end{aligned}
$$

where $\mathcal{R}_{m}=r_{m} / L_{D}$. In the limit $\varepsilon \rightarrow \infty$ and $p \rightarrow \infty$ such that $\varepsilon / p$ remains finite, $J(t)$ becomes the constant current density $\sigma_{c}[\varepsilon /(\pi p)]$. In the limit $R_{m} \rightarrow 0$, the total current density becomes $J(t)=\sigma_{c} E_{\text {ext }}(t)$. It follows from Eq. (24) that the integral of the total current density $J(t)$ over all time is determined by $\sigma_{c} E_{\text {ext }}(t)$ only

$$
\int_{-\infty}^{\infty} J(t) d t=\sigma_{c} \varepsilon
$$

The total amount of electronic charge flowing through the wire is a constant $Q=\pi r_{m}^{2} \sigma_{c} \varepsilon$, irrespective of the width $2 p$ of the pulse, because the contributions of the induced currents average to zero. If $Q$ is the total charge stored in a bank of capacitors, the discharge of which provides the current pulse, then this relation may be taken as a definition of $\varepsilon$ :

$$
\varepsilon=\frac{Q}{\pi r_{m}^{2} \sigma_{c}}=Q \kappa .
$$

In this equation, we see that $\varepsilon$ is dependent on the resistance per unit length, $\kappa$, of the wire as well as the charge $Q$ stored in the bank of capacitors. Electromagnetic induction changes the rate and even the direction of the net flow of electronic charge in a complex manner that depends on the radius and time. At each radius the induced current must reverse the direction of its flow at certain times to satisfy Eq. (24). In Fig. 4, we plot $J(\mathcal{T})$ given by Eq. (40) as a function of $\mathcal{R}_{m}$ and $\mathcal{T}$.

Using Eqs. (9), (31), and (36), we obtain the following expression for the Lorentz force due to the Lorentzian pulse:

$$
\begin{aligned}
\mathbf{f}_{L}(r, t) & =-\frac{\sigma_{c} \varepsilon^{2}}{\pi^{2} r} \frac{\exp \left[-\mu \sigma_{c} r^{2} t /\left(2\left(p^{2}+t^{2}\right)\right)\right]}{\sqrt{p^{2}+t^{2}}}\left(\sin \left[\frac{\mu \sigma_{c} r^{2} p}{2\left(p^{2}+t^{2}\right)}+\tan ^{-1}\left(\frac{t}{p}\right)\right]-\frac{t}{\sqrt{t^{2}+p^{2}}}\right) \hat{\mathbf{r}}, \\
& =-\frac{\sigma_{c}(\varepsilon / \pi p)^{2}}{2} 2 p \frac{1}{L_{D}} \frac{\exp \left[-2 \mathcal{R}^{2} \mathcal{T} /\left(1+4 \mathcal{T}^{2}\right)\right]}{\mathcal{R} \sqrt{1+4 \mathcal{T}^{2}}}\left(\sin \left(\frac{\mathcal{R}^{2}}{\left(1+4 \mathcal{T}^{2}\right)}+\tan ^{-1}(2 \mathcal{T})\right)-\frac{2 \mathcal{T}}{\sqrt{1+4 \mathcal{T}^{2}}}\right) \hat{\mathbf{r}} .
\end{aligned}
$$




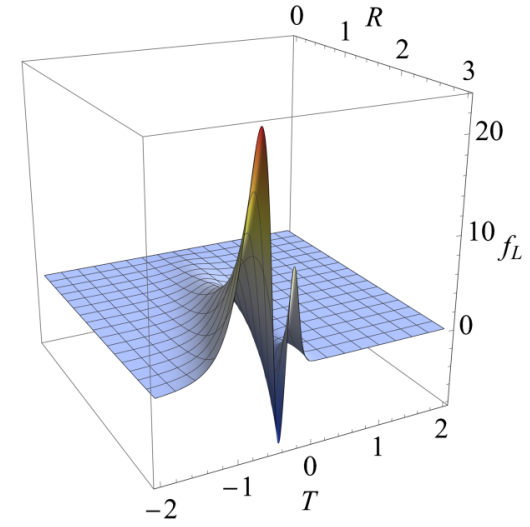

FIG. 5. Plot of the Lorentz force $f_{L}(\mathcal{R}, \mathcal{T})$ in a cylindrical wire due to a current pulse according to Eq. (44). The Lorentz force $f_{L}$ is in units of $\left(\sigma_{c}(\varepsilon / \pi p)^{2} / 2\right) 2 p / L_{D}$.

In this equation, we see the Lorentz force per unit volume is proportional to $\sigma_{c}(\varepsilon / \pi p)^{2} / 2 \times 2 p$ divided by the diffusion length $L_{D}$. In the limit $\varepsilon \rightarrow \infty$ and $p \rightarrow \infty$ such that $\varepsilon / p$ remains finite the Lorentz force becomes $\mu j^{2} r / 2$, where $j=$ $\sigma_{c}[\varepsilon /(\pi p)]$, in agreement with Eq. (E2). Figure 5 is a plot of the Lorentz force according to Eq. (44). Once again we see a steep rise with increasing $\mathcal{R}$. At $\mathcal{R}=3$, the Lorentz force changes sign before $\mathcal{T}=0$.

The stresses created by these Lorentz forces are calculated in the next section.

\section{Time-dependent stress field created by a Lorentzian current pulse}

It was shown in Sec. IV B that the Lorentz forces on conduction electrons are transmitted to ions as body forces. They create stresses which vary with time, because the Lorentz forces are also time dependent. This raises the question of whether the stresses should be calculated elastodynamically, that is whether an inertia term should be included in Eq. (E5).
The stresses are radial and azimuthal and they create longitudinal elastic waves that propagate radially at a speed $c_{l}$. The duration of the Lorentzian pulse is approximately $2 p$ and the distance traveled by a longitudinal wave in this time is $2 c_{l} p$. Since $c_{l}$ is of order $10^{3} \mathrm{~ms}^{-1}$ and $2 p$ is of order $10^{-4} \mathrm{~s}$, then $2 c_{l} p$ is of order $10 \mathrm{~cm}$. This is much larger than the radii of the wires considered experimentally. It is therefore unnecessary to include inertia terms in the calculation of the elastic response of the wire to the Lorentz forces. The duration of the pulses would have to be about $1 \mu \mathrm{s}$, or $r_{m}$ would have to be larger than $\approx 10 \mathrm{~cm}$, for an elastodynamic treatment to be necessary.

We calculate the elastic displacement field $u(r, t)$ using Eq. (E5) but with the Lorentz force $-\mu j_{0}^{2} r / 2$ replaced by the Lorentz force $f_{L}(r, t)$ of Eq. (43):

$$
u^{\prime \prime}+\frac{u^{\prime}}{r}-\frac{u}{r^{2}}=-\frac{(1-2 v)}{2(1-v)} \frac{f_{L}(r, t)}{G_{m}} .
$$

The boundary conditions are that the radial displacement is zero at $r=0$ and $\sigma_{r r}\left(r_{m}, t\right)=0$ for all $t$. The solution may be expressed in terms of the time-independent Green's function $G\left(r, r_{1}\right)$ of the differential equation:

$$
u(r, t)=-\frac{(1-2 v)}{2(1-v)} \frac{1}{G_{m}} \int_{r_{1}=0}^{r_{m}} G\left(r, r_{1}\right) f_{L}\left(r_{1}, t\right) d r_{1} .
$$

where the Green's function is constructed to satisfy the two boundary conditions. The Green's function is as follows:

$$
G\left(r, r_{1}\right)=\left\{\begin{array}{ll}
-\frac{1}{2}\left((1-2 v) r r_{1}^{2} / r_{m}^{2}+r_{1}^{2} / r\right) & 0 \leqslant r_{1} \leqslant r \leqslant r_{m} \\
-\frac{1}{2}\left((1-2 v) r r_{1}^{2} / r_{m}^{2}+r\right) & 0 \leqslant r \leqslant r_{1} \leqslant r_{m}
\end{array} .\right.
$$

We have verified that when this Green's function is inserted in the integral in Eq. (46) with the Lorentz force $-\mu j_{0}^{2} r / 2$ the displacement field reproduces the solution given in Eqs. (E6) and (E7) for a constant current flowing. The strains $e_{r r}(r, t)=$ $u^{\prime}(r, t)$ and $e_{\phi \phi}=u(r, t) / r$ are substituted into Hooke's law of Eq. (E4) to obtain the following expressions for the stresses induced in the wire by the current pulse:

$$
\begin{gathered}
\sigma_{r r}(r, t)=-\int_{r_{1}=0}^{r_{m}} \frac{\partial G\left(r, r_{1}\right)}{\partial r} f_{L}\left(r_{1}, t\right) d r_{1}-\frac{v}{1-v} \frac{1}{r} \int_{r_{1}=0}^{r_{m}} G\left(r, r_{1}\right) f_{L}\left(r_{1}, t\right) d r_{1}, \\
\sigma_{\phi \phi}(r, t)=-\frac{v}{1-v} \int_{r_{1}=0}^{r_{m}} \frac{\partial G\left(r, r_{1}\right)}{\partial r} f_{L}\left(r_{1}, t\right) d r_{1}-\frac{1}{r} \int_{r_{1}=0}^{r_{m}} G\left(r, r_{1}\right) f_{L}\left(r_{1}, t\right) d r_{1}, \\
\sigma_{z z}(r, t)=-\frac{v}{1-v} \int_{r_{1}=0}^{r_{m}}\left(\frac{1}{r} G\left(r, r_{1}\right)+\frac{\partial G\left(r, r_{1}\right)}{\partial r}\right) f_{L}\left(r_{1}, t\right) d r_{1} .
\end{gathered}
$$

We shall now give the full expressions for these stresses and plot them. We obtain the following expression for $\sigma_{z z}$ in terms of the dimensionless variables $\mathcal{R}=r / L_{D}, \mathcal{R}_{m}=r_{m} / L_{D}$, and $\mathcal{T}=t /(2 p)$ :

$$
\begin{aligned}
\frac{\sigma_{z z}(\mathcal{R}, \mathcal{T})}{\left[\sigma_{c}(\varepsilon / \pi p)^{2} p\right]}= & -\frac{v(1-2 v)}{1-v} \frac{1}{\mathcal{R}_{m}^{2}} \sin ^{2}\left(\frac{R_{m}^{2}}{2\left(1+4 \mathcal{T}^{2}\right)}\right) \exp \left[-\frac{2 \mathcal{R}_{m}^{2} \mathcal{T}}{1+4 \mathcal{T}^{2}}\right] \\
& -\frac{v}{2(1-v)} \frac{1}{\left(1+4 \mathcal{T}^{2}\right)}\left[\operatorname{Re}\left\{(2 \mathcal{T}+i) \operatorname{Ei}\left(\frac{-\mathcal{R}_{m}^{2}}{2 \mathcal{T}-i}\right)\right\}-2 \mathcal{T} \operatorname{Ei}\left(\frac{-2 \mathcal{T}_{m}^{2}}{1+4 \mathcal{T}^{2}}\right)\right. \\
& \left.-\operatorname{Re}\left\{(2 \mathcal{T}+i) \operatorname{Ei}\left(\frac{-\mathcal{R}^{2}}{2 \mathcal{T}-i}\right)\right\}+2 \mathcal{T} \operatorname{Ei}\left(\frac{-2 \mathcal{T} \mathcal{R}^{2}}{1+4 \mathcal{T}^{2}}\right)\right],
\end{aligned}
$$



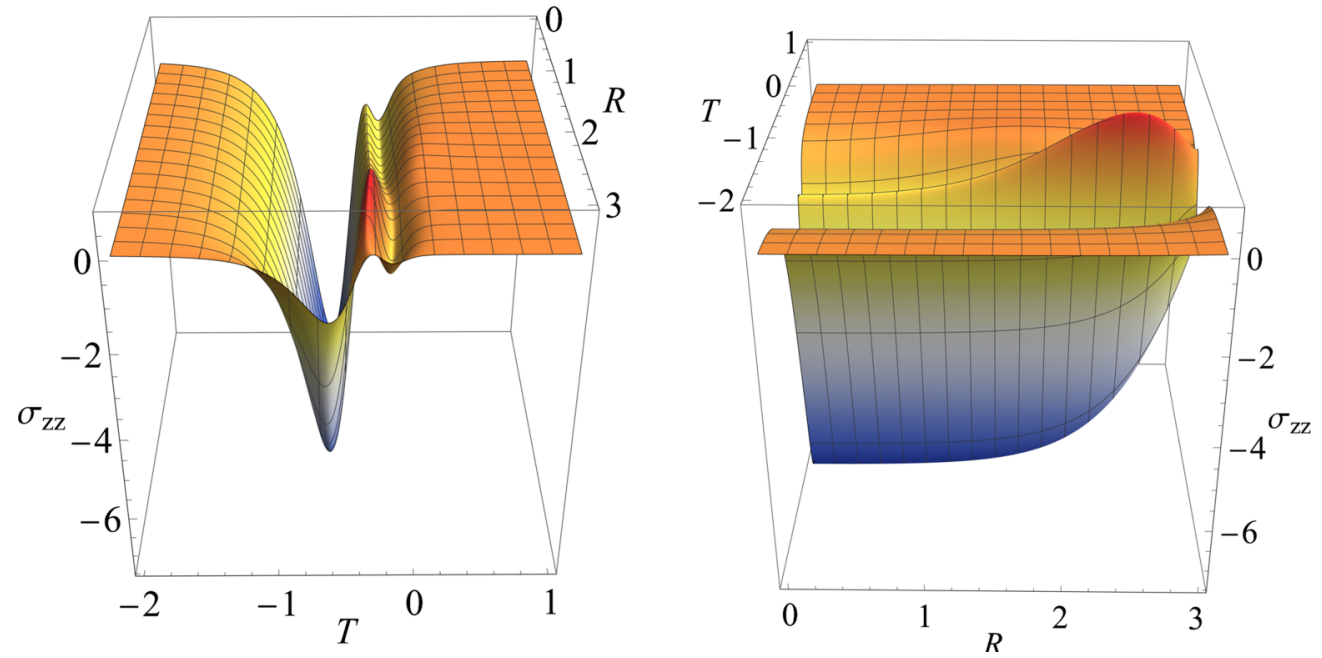

FIG. 6. Views of the stress component $\sigma_{z z}(\mathcal{R}, \mathcal{T})$, given by Eq. (51), for the dimensionless radius $0 \leqslant \mathcal{R} \leqslant 3$ and the dimensionless time $-2 \leqslant \mathcal{T} \leqslant 1$ with $\mathcal{R}_{m}=3$ and $v=1 / 3$. (Left) View from the surface of the wire (at $\mathcal{R}=\mathcal{R}_{m}=3$ ) looking towards the center (at $\mathcal{R}=0$ ). (Right) Side view with the wire center on the left and the wire surface on the right. $\sigma_{z z}$ is expressed in units of $\sigma_{c}(\varepsilon / \pi p)^{2} p=Q^{2} /\left(\pi^{4} r_{m}^{4} \sigma_{c} p\right)$.

where $\operatorname{Ei}(\zeta)$ is the exponential integral function:

$$
\operatorname{Ei}(\zeta)=-\mathrm{P} \int_{-\zeta}^{\infty} \frac{e^{-t}}{t} d t
$$

and $\mathrm{P}$ denotes the principal value. If $\sigma_{z z}(\mathcal{R}, \mathcal{T})$ is expanded to lowest order in $\mathcal{R}$ and $\mathcal{R}_{m}$ it should reduce to the stress $\sigma_{z z}$ of Eq. (E10) when there is a constant direct current density. It may be verified that this is the case if $j_{0}$ of Eq. (E10) is identified with the maximum current density delivered by the pulse, which is equal to $\sigma_{c} \varepsilon /(\pi p)$ and which occurs when $t=$ $\mathcal{T}=0$. This provides some confidence in Eq. (51). The unit of stress in Eq. (51) and subsequent stresses is $\sigma_{c}(\varepsilon / \pi p)^{2} p=$ $Q^{2} /\left(\pi^{4} r_{m}^{4} \sigma_{c} p\right)$, where we have used Eq. (42).

Figure 6 shows a plot of $\sigma_{z z}(\mathcal{R}, \mathcal{T})$ in a wire with $\mathcal{R}_{m}=3$ and $v=1 / 3$. As in earlier plots, it is seen in the left-hand image that most of the interesting features occur when $t \leqslant 0$. The stress is predominantly compressive, as seen in the image on the right. The requirement that the radial stress $\sigma_{r r}$ is zero at the surface (at $\mathcal{R}=\mathcal{R}_{m}=3$ ) at all times has a marked influence on $\sigma_{z z}$. The current density $\sigma_{c} \varepsilon / \pi p$ may be identified with the peak current density only in the limit of $\mathcal{R}_{m}<1$. When $\mathcal{R}_{m}>1$, it is more useful to express $\sigma_{c} \varepsilon / \pi p$ in terms of the total charge $Q$ flowing through the wire during the pulse, using Eq. (42): $\sigma_{c} \varepsilon / \pi p=Q /\left(\pi^{2} r_{m}^{2} p\right)$. For a current pulse with $p=30 \mu \mathrm{s}, Q=7 \mathrm{C}$ (corresponding to charging a $10 \mathrm{mF}$ capacitor bank to $700 \mathrm{~V}$ ), in a copper wire with $v=1 / 3$, and $r_{m}=2.7 \mathrm{~mm}$ (corresponding to $\left.\mathcal{R}_{m}=3\right)$, we find $Q /\left(\pi^{2} r_{m}^{2} p\right) \approx 3 \times 10^{9} \mathrm{~A} \mathrm{~m}^{-2}$, the maximum compressive stress $\sigma_{z z}$ is around $32 \mathrm{MPa}$. If $r_{m}=$ $0.5 \mathrm{~mm}$, corresponding to $\mathcal{R}_{m}=0.6$ and $Q=0.2 \mathrm{C}$ so that $Q /\left(\pi^{2} r_{m}^{2} p\right)$ is again $3 \times 10^{9} \mathrm{~A} \mathrm{~m}^{-2}$, the maximum compressive stress decreases to approximately $0.4 \mathrm{MPa}$, in agreement with the estimate in Sec. E.

Consider a wire of BCC iron $(v \approx 0.3)$ with a radius of $0.3 \mathrm{~mm}$, the same value of $Q /\left(\pi^{2} r_{m}^{2} p\right)$, and assuming the most conservative value for $L_{D}$ of $70 \mu \mathrm{m}$, we obtain $\mathcal{R}_{m}=4.3$. The stress $\sigma_{z z}$ changes from compression to tension twice and then returns to compression again in less than $60 \mu \mathrm{s}$. The maximum compressive stress is approximately $9 \mathrm{GPa}$ and the maximum tensile stress is approximately $1 \mathrm{GPa}$. This demonstrates the strong effect of a large magnetic permeability on the induced stresses.

The radial stress $\sigma_{r r}(\mathcal{R}, \mathcal{T})$ is similarly expressed as follows:

$$
\begin{aligned}
\frac{\sigma_{r r}(\mathcal{R}, \mathcal{T})}{\left[\sigma_{c}(\varepsilon / \pi p)^{2} p\right]}= & \frac{1-2 v}{2(1-v)}\left[\frac{1}{\mathcal{R}^{2}} \sin ^{2}\left(\frac{\mathcal{R}^{2}}{2\left(1+4 \mathcal{T}^{2}\right)}\right) \exp \left(-\frac{2 \mathcal{R}^{2} \mathcal{T}}{1+4 \mathcal{T}^{2}}\right)-\frac{1}{\mathcal{R}_{m}^{2}} \sin ^{2}\left(\frac{\mathcal{R}_{m}^{2}}{2\left(1+4 \mathcal{T}^{2}\right)}\right) \exp \left(-\frac{2 \mathcal{R}_{m}^{2} \mathcal{T}}{1+4 \mathcal{T}^{2}}\right)\right] \\
& -\frac{1}{4(1-v)} \frac{1}{\left(1+4 \mathcal{T}^{2}\right)}\left[\operatorname{Re}\left\{(2 \mathcal{T}+i) \operatorname{Ei}\left(\frac{-\mathcal{R}_{m}^{2}}{2 \mathcal{T}-i}\right)\right\}-2 \mathcal{T} \operatorname{Ei}\left(\frac{-2 \mathcal{T} \mathcal{R}_{m}^{2}}{1+4 \mathcal{T}^{2}}\right)\right. \\
& \left.-\operatorname{Re}\left\{(2 \mathcal{T}+i) \operatorname{Ei}\left(\frac{-\mathcal{R}^{2}}{2 \mathcal{T}-i}\right)\right\}+2 \mathcal{T} \operatorname{Ei}\left(\frac{-2 \mathcal{T} \mathcal{R}^{2}}{1+4 \mathcal{T}^{2}}\right)\right]
\end{aligned}
$$

Figure 7 shows plots of $\sigma_{r r}(\mathcal{R}, \mathcal{T})$, assuming $v=1 / 3$ and $\mathcal{R}_{m}=3$. It is quite similar to $\sigma_{z z}(\mathcal{R}, \mathcal{T})$, as expected, but $\sigma_{r r}(\mathcal{R}, \mathcal{T})=0$ at all times at the surface of the wire as required by the boundary condition. Equation (53) reduces to Eq. (E8) in the limit of small $\mathcal{R}$ and $\mathcal{R}_{m}$ provided $j_{0}$ is identified with $\sigma_{c} \varepsilon /(\pi p)$ and $\mathcal{T}$ is set to zero. 

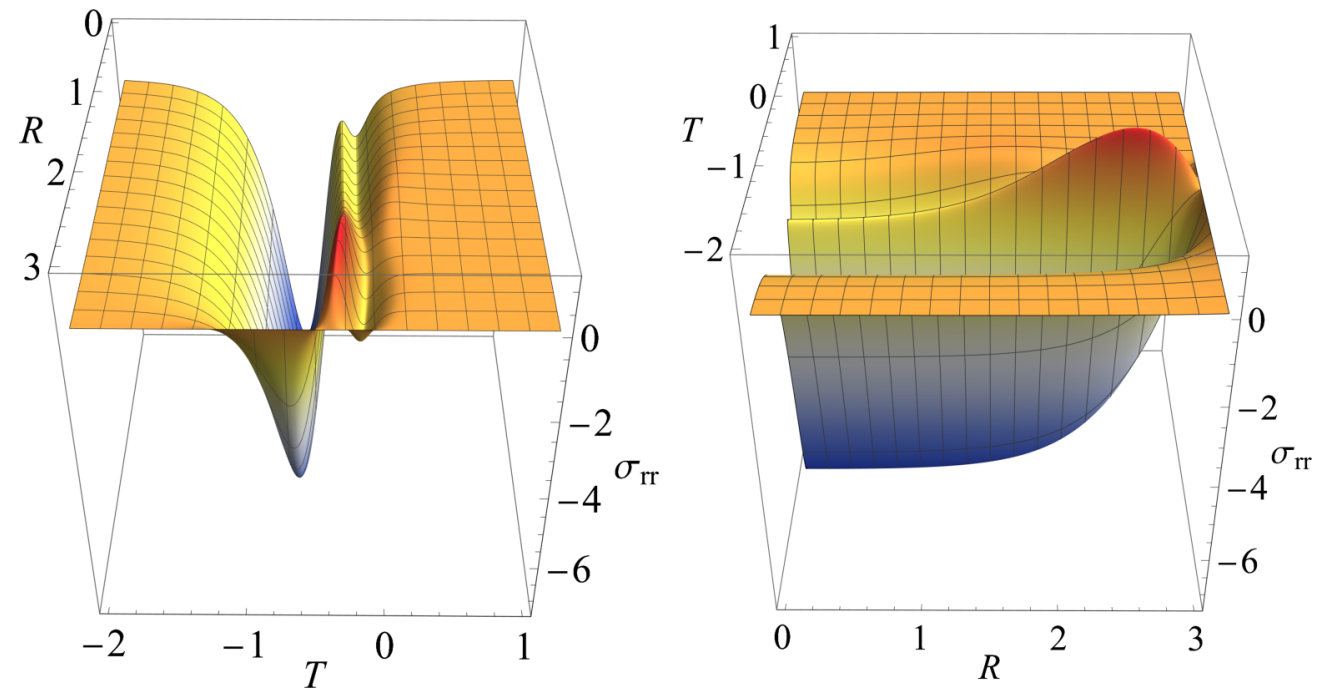

FIG. 7. Views of the stress component $\sigma_{r r}(\mathcal{R}, \mathcal{T})$, given by Eq. (53), for the dimensionless radius $0 \leqslant \mathcal{R} \leqslant 3$ and the dimensionless time $-2 \leqslant \mathcal{T} \leqslant 1$, with $\mathcal{R}_{m}=3$ and $v=1 / 3$. (Left) View from the wire surface $(\mathcal{R}=3$ ) looking towards the center of the wire $(\mathcal{R}=0)$. (Right) Side view with the wire center on the left and the wire surface on the right. $\sigma_{r r}$ is expressed in units of $\sigma_{c}(\varepsilon / \pi p)^{2} p=Q^{2} /\left(\pi^{4} r_{m}^{4} \sigma_{c} p\right)$.

The hoop stress $\sigma_{\phi \phi}$ is as follows:

$$
\begin{aligned}
\frac{\sigma_{\phi \phi}(\mathcal{R}, \mathcal{T})}{\left[\sigma_{c}(\varepsilon / \pi p)^{2} p\right]}= & -\frac{1-2 v}{2(1-v)}\left[\frac{1}{\mathcal{R}^{2}} \sin ^{2}\left(\frac{\mathcal{R}^{2}}{2\left(1+4 \mathcal{T}^{2}\right)}\right) \exp \left(-\frac{2 \mathcal{R}^{2} \mathcal{T}}{1+4 \mathcal{T}^{2}}\right)+\frac{1}{\mathcal{R}_{m}^{2}} \sin ^{2}\left(\frac{\mathcal{R}_{m}^{2}}{2\left(1+4 \mathcal{T}^{2}\right)}\right) \exp \left(-\frac{2 \mathcal{R}_{m}^{2} \mathcal{T}}{1+4 \mathcal{T}^{2}}\right)\right] \\
& -\frac{1}{4(1-v)} \frac{1}{\left(1+4 \mathcal{T}^{2}\right)}\left[\operatorname{Re}\left\{(2 \mathcal{T}+i) \operatorname{Ei}\left(\frac{-\mathcal{R}_{m}^{2}}{2 \mathcal{T}-i}\right)\right\}-2 \mathcal{T} \operatorname{Ei}\left(\frac{-2 \mathcal{T} \mathcal{R}_{m}^{2}}{1+4 \mathcal{T}^{2}}\right)\right. \\
& \left.-\operatorname{Re}\left\{(2 \mathcal{T}+i) \operatorname{Ei}\left(\frac{-\mathcal{R}^{2}}{2 \mathcal{T}-i}\right)\right\}+2 \mathcal{T} \operatorname{Ei}\left(\frac{-2 \mathcal{T} \mathcal{R}^{2}}{1+4 \mathcal{T}^{2}}\right)\right]
\end{aligned}
$$

We have verified that Eqs. (51), (53) and (54) satisfy $\sigma_{z z}(\mathcal{R}, \mathcal{T})=v\left[\sigma_{r r}(\mathcal{R}, \mathcal{T})+\sigma_{\phi \phi}(\mathcal{R}, \mathcal{T})\right]$, as required in plane strain. Equation (54) reduces to Eq. (E9) in the limit of small $\mathcal{R}$ and $\mathcal{R}_{m}$ provided $j_{0}$ is identified with $\sigma_{c} \varepsilon /(\pi p)$ and $\mathcal{T}$ is set to zero. Figure 8 shows a plot of $\sigma_{\phi \phi}(\mathcal{R}, \mathcal{T})$ according to Eq. (54).

As we have the full stress tensor induced by the current pulse it is possible to calculate the von Mises shear stress
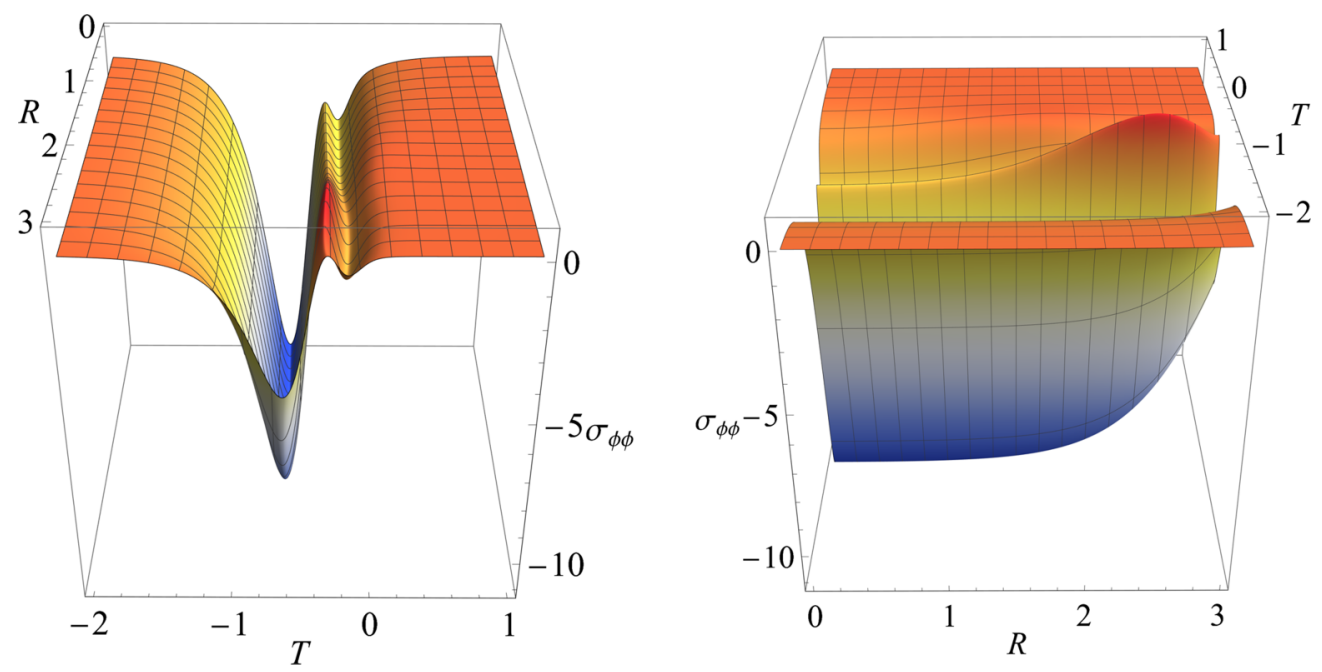

FIG. 8. Views of the stress component $\sigma_{\phi \phi}(\mathcal{R}, \mathcal{T})$, given by Eq. (54), for the dimensionless radius $0 \leqslant \mathcal{R} \leqslant 3$ and the dimensionless time $-2 \leqslant \mathcal{T} \leqslant 1$, with $\mathcal{R}_{m}=3$ and $v=1 / 3$. (Left) View from the wire surface $(\mathcal{R}=3$ ) looking towards the surface of the wire $(\mathcal{R}=0)$. (Right) Side view with the wire center on the left and the wire surface on the right. $\sigma_{\phi \phi}$ is expressed in units of $\sigma_{c}(\varepsilon / \pi p)^{2} p=Q^{2} /\left(\pi^{4} r_{m}^{4} \sigma_{c} p\right)$. 


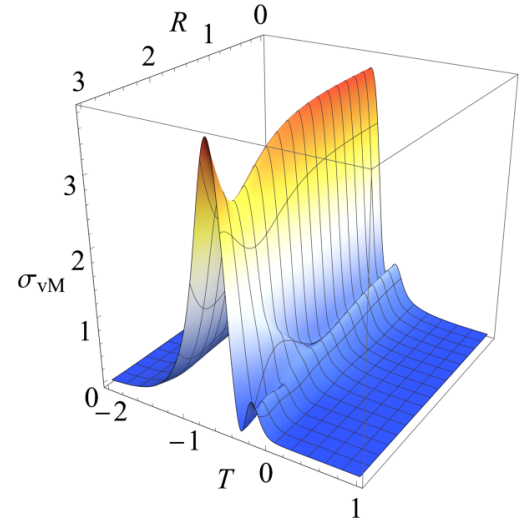

FIG. 9. Plot of the von Mises shear stress, given by Eq. (55), assuming $v=1 / 3$ and $\mathrm{R}_{m}=3$. The von Mises stress is expressed in units of $\sigma_{c}(\varepsilon / \pi p)^{2} p=Q^{2} /\left(\pi^{4} r_{m}^{4} \sigma_{c} p\right)$.

invariant. It is plotted in Fig. 9. The von Mises stress is defined as follows:

$$
\sigma_{v M}=\frac{1}{\sqrt{2}} \sqrt{\left(\sigma_{z z}-\sigma_{r r}\right)^{2}+\left(\sigma_{r r}-\sigma_{\phi \phi}\right)^{2}+\left(\sigma_{\phi \phi}-\sigma_{z z}\right)^{2}} .
$$

The root mean square shear stress averaged over all planes is equal to $\sqrt{2 / 15} \sigma_{v M}$ [24]. The shear stresses $\left(\sigma_{z z}-\sigma_{\phi \phi}\right) / 2$ and $\left(\sigma_{z z}-\sigma_{r r}\right) / 2$ are plotted in Fig. 10. They act on planes inclined at $45^{\circ}$ to the wire axis and they promote dislocation glide. When the magnitude of these shear stress pulses is sufficient to liberate dislocations from pinning centers we suggest this is the mechanism by which current pulses lead to the enhanced plasticity observed in the electroplastic effect.

\section{DISCUSSION AND CONCLUSIONS}

Each of the four mechanisms of the electroplastic effect discussed in Appendices B-E assumes the pulsing of the current plays no role other than to minimize Joule heating. Consequently, each of these mechanisms is equally applicable to a continuous direct current as to a pulsed direct current. In contrast, the focus of this paper has been the influence of pulsing the current on the generation of stresses resulting from electromagnetic induction. Glide of dislocations and liberation of dislocations from pinning points requires appropriate stresses. With intense current pulses lasting between 10 and $100 \mu \mathrm{s}$ there is the possibility of larger stresses created by electromagnetic induction than those arising when the current is continuous. Our thinking was inspired in part by the technology of electromagnetic forming (for a review see Ref. [25]). In this technology magnetic fields associated with pulses of large current densities in a solenoid induce currents in a workpiece. As first demonstrated by Kapitza [26] almost a century ago, the induced currents flowing in the workpiece in the magnetic field of the adjacent solenoid create forces in the workpiece which deform it plastically at a high strain rate. The question we addressed in this paper is whether the currents induced by the temporal variation of the magnetic field inside the wire can generate stresses significantly above those of a continuous current. The short answer is yes under certain conditions.

The parameter $L_{D}=\sqrt{2 p /\left(\mu \sigma_{c}\right)}$ is the characteristic length in the vector diffusion equation, Eq. (6), governing the evolution in space and time of the current density in an infinitely long, homogeneous, cylindrical wire of radius $r_{m}$. The same vector diffusion equation applies to the induced electric field and the magnetic field. The parameter $L_{D}$ is the same as the skin depth of a conductor carrying an alternating current of angular frequency $1 / p$. When $\mathcal{R}_{m}=r_{m} / L_{D}$ is less than 1 the effects of electromagnetic induction of the current density are small. The current density is then adequately described by $j(r, t)=\sigma_{c} E_{\text {ext }}(t)$, i.e., the radial dependence is negligible. The dynamic pinch effect is then virtually indistinguishable from the static pinch effect. The condition $\mathcal{R}_{m}>1$ encapsulates when the effects of electromagnetic induction are significant. Larger values of $\mathcal{R}_{m}$ arise when the wire radius $r_{m}$ increases, the pulse width $2 p$ decreases, or the wire has a larger electrical conductivity $\sigma_{c}$ or larger magnetic permeability $\mu$.

The question arises whether $\mathcal{R}_{m}$ can be varied without affecting the unit of stress in which all the stress components are expressed. This is answered most simply by rewriting the unit of stress, $\sigma_{c}(\varepsilon / \pi p)^{2} p$, as $Q^{2} /\left(\pi^{4} r_{m}^{4} \sigma_{c} p\right)$, using Eq. (42). Since $\mathcal{R}_{m}=r_{m} \sqrt{\mu \sigma_{c} /(2 p)}$ the only way to change $R_{m}$ without affecting the unit of stress is to vary the magnetic permeability because the unit of stress is independent of $\mu$. However, it is difficult in practice to vary the permeability without also affecting the conductivity. Nevertheless $\mu$ can be varied by alloying and/or heat treatment [23], while adjusting $Q$ to compensate for the change in $\sigma_{c}$ to maintain the unit of stress constant. This would permit an experimental test of the predicted strong dependence of the stress drops on magnetic permeability.

Figure 3 of Okazaki et al. [10] shows the stress drops as a function of current density in $99.9 \%$ iron wires of $0.35 \mathrm{~mm}$ diameter and in $99.97 \%$ titanium wires of diameters 1.31 and $1.36 \mathrm{~mm}$ diameter. They found the stress drops in the iron wire are about an order of magnitude greater than in the titanium wires at current densities between $5 \times 10^{8}$ and $10 \times 10^{8}$ $\mathrm{A} \mathrm{m}^{-2}$. This observation is consistent with the smaller value of $L_{D}$ in iron compared with titanium, raising the value of $\mathcal{R}_{m}$ in the iron wire despite the smaller value of $r_{m}$. Such a large difference between the stress drops in iron and titanium cannot be explained by any other proposed mechanism.

For a given metal the static pinch effect predicts, at constant current density, that the average stress $\left\langle\sigma_{z z}\right\rangle$ is proportional to $r_{m}^{2}$. The dynamic pinch effect predicts a much stronger dependence of the peak stress on $r_{m}$ as $\mathcal{R}_{m}$ increases above one, while maintaining the unit of stress $Q^{2} /\left(\pi^{4} r_{m}^{4} \sigma_{c} p\right)$ constant. This can be tested experimentally.

The Lorentz force on conduction electrons is conveyed as a body force to the ions of the crystal lattice by the electron-ion interaction. This body force generates mechanical stresses through the equation of mechanical equilibrium, Eq. (E3). Pulsing the current creates body force pulses, which in turn generate elastic waves. Mechanical vibrations have been detected experimentally by applying piezoelectric sensors to the surface of solid cylindrical or tubular conductors subjected to current pulses [27]. 

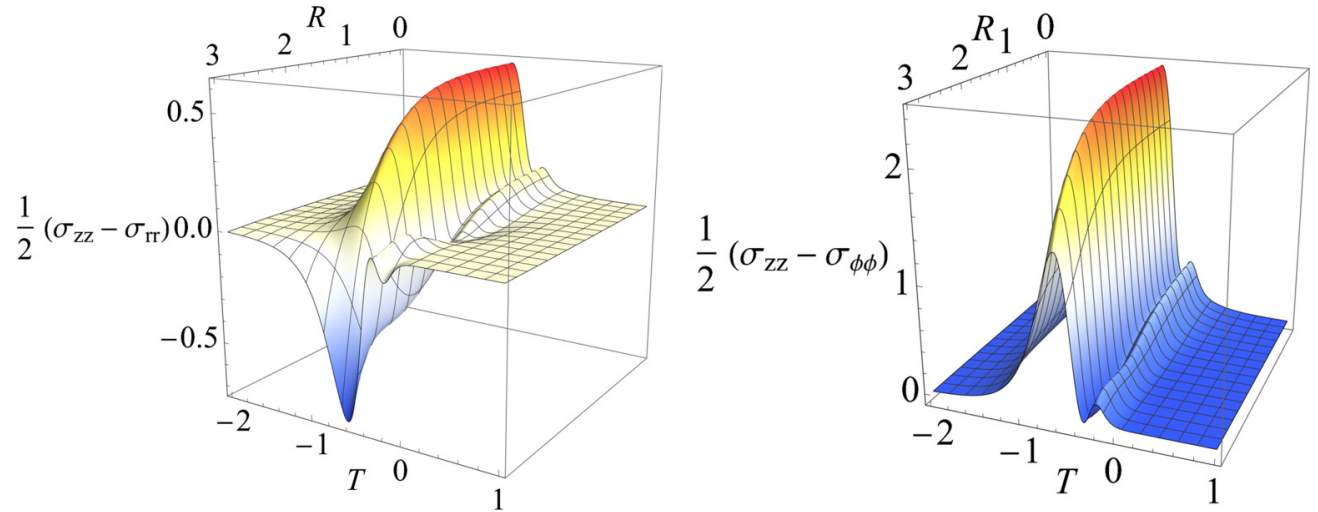

FIG. 10. Shear stress components $\left(\sigma_{z z}(\mathcal{R}, \mathcal{T})-\sigma_{r r}(\mathcal{R}, \mathcal{T})\right) / 2$ (left) and $\left(\sigma_{z z}(\mathcal{R}, \mathcal{T})-\sigma_{\phi \phi}(\mathcal{R}, \mathcal{T})\right) / 2$ (right) for the dimensionless radius $0 \leqslant \mathcal{R} \leqslant 3$ and the dimensionless time $-2 \leqslant \mathcal{T} \leqslant 1$, with $\mathcal{R}_{m}=3$ and $\nu=1 / 3$. The stresses are expressed in units of $\sigma_{c}(\varepsilon / \pi p)^{2} p=$ $Q^{2} /\left(\pi^{4} r_{m}^{4} \sigma_{c} p\right)$.

The assumption of a homogeneous wire was necessary to be able to solve the equations analytically. In practice there are bound to be microstructural inhomogeneities, from point defects to precipitates, dislocations, slip bands, twins and grain boundaries. These features will affect the distribution of the current density and hence the magnetic field and Lorentz forces. The assumption of elastic isotropy is also an approximation in all metals, although pure tungsten is very close to being isotropic elastically. In elastically anisotropic polycrystalline wires, current pulses may well create transient compatibility stresses at grain boundaries, in addition to and comparable to the transient stresses created directly by current pulses.

Finally, we note that the stresses we have calculated in this paper provide a mechanism of current-induced mass transport separate from and in addition to the usual mechanism of electromigration. Gradients in elastic strains associated with spatially varying current-induced stresses give rise to additional forces on point defects through the interaction with their dipole tensors [24]. Whereas the origin of this force is long range, spatially varying elastic fields, the origin of the electromigration force is local, arising from the self-consistent redistribution of electronic charge at the point defect in the presence of the current [28-31].

In conclusion, we contend that significantly larger transient stresses can be generated by pulsing a current than the constant stresses arising when the current is constant in time. The effect of pulsing the current becomes significant when $\mathcal{R}_{m}>1$, most notably when the current changes rapidly and in larger samples and in magnetic materials. We suggest that these transient stresses are responsible for the stress drops observed when the metal wire is stretched elastically. They also alter the stress-state of the metal when it is deforming plastically, although the response of the metal is then more complex. The shape of the current pulse is important because it determines how rapidly the current rises and falls. For example, in an applied current pulse that approximates to a square wave, the steep rise and fall of the applied current may lead to much larger induced currents than the maximum of the applied current. The induced currents change direction during an applied current pulse, such that their average over time is zero. However, the Lorentz body forces and the stresses they create are independent of the direction of the induced currents and they remain finite.

After completing this research we became aware of a short paper by Bataronov and Roshchupkin [32] which also considered a dynamic version of the pinch effect as a possible explanation of electroplasticity. Their theory is also mentioned in Ref. [33]. Although the basic idea appears to be the same their mathematical analysis is completely different. The expression they state for the induced current density during a current pulse does not appear to satisfy our Eq. (24). We do not understand their analysis.

\section{ACKNOWLEDGMENTS}

A.P.S. is grateful to Rongshan Qin who first drew his attention to electroplasticity sixteen years ago. T.N.T. is grateful to Andrew Horsfield for many discussions about electroplasticity.

\section{APPENDIX A: LIST OF SYMBOLS}

\begin{tabular}{ll}
\hline \hline List of symbols \\
\hline $\mathbf{A}$ & magnetic vector potential \\
$\alpha(\omega)$ & amplitude of $\bar{j}(r, \omega)$ \\
$\mathbf{b}$ & Burgers vector \\
$\mathbf{B}$ & magnetic field \\
$B$ & coefficient of dislocation drag \\
$B_{e}$ & electronic contribution to coefficient of dislocation drag \\
$B_{p}$ & phonon contribution to coefficient of dislocation drag \\
$\bar{B}(r, \omega)$ & Fourier transform of $B(r, t)$ \\
$c$ & speed of light \\
$c_{l}$ & speed of longitudinal elastic waves \\
$C_{1}, C_{2}$ & arbitrary constants of integration \\
$\delta(\omega)$ & Dirac delta function \\
$\delta \rho_{0}$ & charge density associated with Hall electric field \\
$\Delta \sigma$ & stress drop \\
$\mathcal{E}$ & Hall electric field \\
$e$ & electronic charge \\
$\mathbf{e}$ & linear elastic strain tensor \\
$\varepsilon$ & parameter in Lorentzian pulse controlling its maximum \\
& for a given value of $p$ \\
$\epsilon_{0}$ & permittivity of free space \\
\hline
\end{tabular}


(Continued.)

\begin{tabular}{|c|c|}
\hline \multicolumn{2}{|c|}{ List of symbols } \\
\hline $\mathbf{E}_{\mathrm{ext}}$ & externally applied electric field \\
\hline $\bar{E}_{\text {ext }}(\omega)$ & Fourier transform of $E_{\text {ext }}(t)$ \\
\hline$\underline{\mathbf{E}_{i}}$ & induced electric field \\
\hline $\bar{E}_{i}(r, \omega)$ & Fourier transform of $E_{i}(r, t)$ \\
\hline$\overline{\mathcal{E}}_{i}(\omega)$ & $\begin{array}{l}\text { Fourier transform of induced electric field averaged over } \\
\text { all radii }\end{array}$ \\
\hline $\mathrm{Ei}$ & exponential integral function \\
\hline$\phi$ & the azimuth in the cylindrical coordinate system \\
\hline$f_{c}$ & $\begin{array}{l}\text { force on a dislocation due to interaction with conduction } \\
\text { electrons }\end{array}$ \\
\hline $\mathbf{F}_{e}$ & net force per unit volume on conduction electrons \\
\hline $\mathbf{F}_{e}^{B}$ & $\begin{array}{l}\text { force per unit volume on conduction electrons due to the } \\
\text { magnetic field }\end{array}$ \\
\hline $\mathbf{F}_{e}^{E}$ & $\begin{array}{l}\text { force per unit volume on conduction electrons due to the } \\
\text { applied electric field and the Hall field }\end{array}$ \\
\hline $\mathbf{F}_{e}^{i}$ & $\begin{array}{l}\text { electrostatic force per unit volume on conduction } \\
\text { electrons due to ions }\end{array}$ \\
\hline $\mathbf{f}_{L}$ & Lorentz force per unit volume \\
\hline $\mathbf{f}_{P K}$ & Peach-Koehler force per unit length on a dislocation \\
\hline$G_{m}$ & elastic shear modulus \\
\hline$G\left(r, r_{1}\right)$ & Green's function for Eq. (45) \\
\hline j & current density \\
\hline$J(t)$ & current density averaged over all radii \\
\hline $\bar{J}(\omega)$ & $\begin{array}{l}\text { Fourier transform of current density averaged over all } \\
\text { radii }\end{array}$ \\
\hline$j_{0}$ & constant current density \\
\hline $\bar{j}(r, \omega)$ & Fourier transform of $j(r, t)$ \\
\hline$J_{0}$ & Bessel function of the first kind of order zero \\
\hline$J_{1}$ & Bessel function of the first kind of order one \\
\hline$j_{i}(r, t)$ & induced current density at radius $r$ and time $t$ \\
\hline$\lambda$ & Lamé's first elastic constant \\
\hline$L_{D}$ & diffusion length \\
\hline$\lambda_{\omega}$ & diffusion length associated with angular frequency $\omega$ \\
\hline$\mu$ & permeability \\
\hline$\mu_{0}$ & permeability of free space \\
\hline$\mu_{r}$ & relative permeability \\
\hline$n$ & number of conduction electrons per unit volume \\
\hline$v$ & Poisson's ratio \\
\hline$p$ & half-width at half maximum of Lorentzian pulse \\
\hline$Q$ & $\begin{array}{l}\text { total amount of electronic charge flowing through the } \\
\text { wire in one current pulse }\end{array}$ \\
\hline$r$ & radial coordinate of the cylindrical coordinate system \\
\hline $\mathcal{R}$ & dimensionless radius $r / L_{D}$ \\
\hline$r_{m}$ & radius of cylindrical wire \\
\hline$\rho_{0}$ & ionic charge density \\
\hline $\mathcal{R}_{m}$ & maximum value of $\mathcal{R}$ equal to $r_{m} / L_{D}$ \\
\hline$\sigma$ & elastic stress tensor \\
\hline$\sigma_{c}$ & electric conductivity \\
\hline$\sigma_{f}$ & flow stress \\
\hline$\sigma_{v M}$ & von Mises shear stress invariant \\
\hline $\mathcal{T}$ & dimensionless time $t /(2 p)$ \\
\hline$\tau_{e}$ & charge relaxation time of conduction electrons \\
\hline$t_{p}$ & duration of current pulse \\
\hline$u$ & radial elastic displacement \\
\hline$v_{d}$ & speed of dislocation \\
\hline$v_{e}$ & drift speed of conduction electrons \\
\hline$\hat{\xi}$ & direction of dislocation line \\
\hline$z$ & coordinate along the axis of the straight cylindrical wire \\
\hline
\end{tabular}

\section{APPENDIX B: DISLOCATION UNPINNING IN A MAGNETIC FIELD}

Dislocations may be pinned by each other through forest interactions or by impurities, surfaces, voids, grain boundaries and precipitates. Molotskii and coworkers proposed that the magnetic field of an electric current may enable the release of dislocations from pinning centers thereby increasing plastic strain [13,17-19]. During an experimental test in which the sample is strained at a constant rate the unpinning of dislocations will result in a reduction $\Delta \sigma$ of the flow stress $\sigma_{f}$.

It appears the pulsing of the current plays no role in this mechanism, other than to minimize the degree of Joule heating. Before we discuss the physical basis of the mechanism proposed by Molotskii et al. we note three of its key features. (1) The mechanism applies to dislocations with arbitrary Burgers vectors and line directions. It enables dislocations to move both with and against the current. (2) The effect of the current on plasticity is dependent on the thermal and mechanical history of the sample, because its history determines the degree of dislocation pinning. (3) Since the mechanism is centered on the unpinning of dislocations it does not apply to the stress drops that have been observed when the sample is deforming purely elastically.

The mechanism proposed by Molotskii and coworkers for the unpinning of dislocations by the magnetic field of the current is as follows. It is assumed that 'dangling bond' states exist in dislocation cores in metals. Point defects are also assumed to exist in the metal such as transition metal impurities and vacancies as well as forest interactions between dislocations. These defects are assumed to be paramagnetic centers with unpaired electron spins. When these paramagnetic point defects are located in the dislocation core they form singlet bonding states or triplet antibonding states with the dangling bonds on metal atoms in the core. If they form singlet bonding states it is more difficult to move the dislocation because they have to move with the core. The dislocation is then pinned. The magnetic field of the current is assumed to favor the triplet states, and since they are antibonding the dislocation is able to escape from the pinning defect. The dislocation is then unpinned. As noted by Molotskii [19], the mechanism can be invoked only when the bond between the dislocation and the point defect is on the verge of breaking. The magnetic field by itself cannot break the bond because that would require an energy of order $0.1-1 \mathrm{eV}$. Although the energy of flipping a spin by the magnetic field is three to four orders of magnitude smaller than this bond energy, and two to three orders of magnitude smaller than a thermal fluctuation at room temperature, once the spin is flipped the bond cannot be reformed.

It is instructive to compare the mechanism proposed by Molotskii et al. to the "radical pair mechanism" invoked to explain the effect of magnetic fields on the rates of chemical reactions in which saturated bonds in molecules are broken [34]. A saturated bond contains two electrons with opposite spins. In the radical pair mechanism when a saturated bond is broken, for example, by an incoming photon, a pair of radicals is produced in which the two electrons retain their opposite spins forming an entangled singlet state. In this state, the saturated bond can be easily reformed by the radicals coming together again. However, if the spin of the unpaired 
electron in one of the radicals is flipped by a magnetic field the probability of the bond being reformed is much reduced. That is because one of the spins would have to be flipped again to form a bonding rather than an antibonding state. In this way, the magnetic field can increase the rate of production of the free radicals. Note that in this mechanism the bond is broken by a photon with an energy of order $1 \mathrm{eV}$. The role of the magnetic field is only to reduce the probability of the bond being reformed, not to break the bond.

In tetrahedrally bonded silicon, which is an insulator at absolute zero, each bond is saturated. Dangling bonds can occur in dislocation cores in the crystalline state, but they are rare because the core reconstructs so that each silicon atom is fourfold coordinated. However, incompatibilities between different reconstructions meeting along the same dislocation line can lead to dangling bond states being formed with energies in the band gap [35]. The dangling bond can contain 0,1 or 2 electrons depending on the position of the Fermi level in the band gap. Thus "dangling bond" states of the type envisaged by Molotskii and coworkers can exist in dislocation cores in silicon.

In contrast to tetrahedrally bonded silicon the bonds in a metal are unsaturated, i.e., they each contain less than two electrons. As a result metallic bonding is more delocalized, and it is more affected by the positions of neighboring atoms. When we speak of a bond between two atoms in a metal being broken it is a more delocalized event and less obvious than when the bonding is saturated. Breaking the bond means the two atoms concerned have separated to the point that the bond order between them has become insignificant. However, that does not result in the formation of two radicals, each containing one electron, localized on the atoms that were previously bonded. Instead, the local densities of states of the two atoms that were bonded, and their neighbors, all adjust to the local change in coordination numbers of the two atoms concerned. As a result there are states which are predominantly weighted along the dislocation core, but electrons in these states are able to leave the core and diffuse into the bulk of the metal. They are virtual bound states rather than bound states. This picture is supported by detailed electronic structure calculations of dislocations in metals and alloys, see, for example, Ref. [36].

For a bound state to exist on a nonmagnetic atom in a metal, it must have an energy outside the conduction band of the metal. Only then can it be decoupled from the itinerant states of the metal. Since the conduction band has no upper limit it must have an energy between the conduction band and the atomic core states of the metal. However, it will then be occupied by two electrons and unable to bond to another atom.

The Anderson model [37] treats the theory of a paramagnetic impurity atom, such as a transition metal atom, in a nonmagnetic metal. Under conditions identified in Anderson's model, the impurity may exist in a localized paramagnetic state within the conduction band of the metal, but the magnetic polarization is generally nonintegral owing to the interaction with conduction electron states of the metal. It is then also in a virtual bound state.

A dangling bond state comprising an unpaired electron confined to a single atom is not a valid concept in a metal. Although the degree of electron localization envisaged by the Molotskii-Fleurov mechanism exists when bonds are saturated, as in silicon, it does not exist in a metal. The theory does not have a sound foundation in metal physics.

\section{APPENDIX C: ELECTROMIGRATION OF DISLOCATIONS}

In the absence of a current, it has been established experimentally that conduction electrons in a metal exert a drag force on moving dislocations. The most convincing experimental evidence comes from measurements made at liquid helium temperatures where phonon drag becomes negligible and drag due to electrons dominates. Kojima and Suzuki [38] found the flow stresses of superconducting niobium and lead increase at the transition between superconducting and normal states. The transition was induced by the application of a magnetic field at $4.2 \mathrm{~K}$. The ratio of the increment to the flow stress in the superconducting state was $2.9 \%$ in lead and $0.6 \%$ in niobium. In the superconducting state, electron drag is quenched, and it appears only in the normal state. There is experimental evidence that screw dislocations experience a smaller electron drag force at a given speed than edge dislocations [39]. One source of electron drag is the atomic restructuring in the core as a dislocation glides, which generates electron-hole pairs in the vicinity of the Fermi energy, thereby dissipating energy. Electron-hole pairs are also created by the motion of the scattering potential the dislocation represents for conduction electrons.

The relation between the drag force $F$ per unit length on a dislocation and its speed $v_{d}$, at low speeds where inertial effects are negligible, is usually expressed as $F=B v_{d}$. The drag coefficient $B$ is usually expressed as $B_{e}+B_{p}$ where $B_{e}$ is the electron contribution and $B_{p}$ is the phonon contribution. The electron contribution dominates only at cryogenic temperatures. Experiments by Hikata et al. [40] found, at temperatures below $40 \mathrm{~K}$, the drag coefficient of dislocations in aluminum is approximately constant at $1.4 \times 10^{-6} \mathrm{sPa}$. They also found that above $40 \mathrm{~K}$ the drag coefficient increases with temperature due to phonon drag.

Viewed in the frame of a dislocation moving through the metal, there is a current of conduction electrons moving past it in the opposite direction. In this reference frame, the drag force is seen as a 'pushing force' acting on a stationary dislocation in the direction of an electron current flowing past it. Antolovich and Conrad [4] identified this pushing force as a current-induced force that leads to electromigration of a stationary dislocation immersed in an electron current. Following earlier work by Kravchenko [41], Klimov et al. [42] and Roshchupkin et al. [43] these authors expressed the force per unit length $f_{c}$ on a dislocation due to interactions with electrons as follows:

$$
f_{c}=B_{e}\left(v_{e}-v_{d}\right),
$$

where $v_{e}$ is the electron drift speed. If the dislocation is stationary $f_{c}$ is the current-induced force $B_{e} v_{e}$. If it is moving at the same speed as the drift speed of the electrons there is no current-induced or electron drag force acting on it. If it is moving faster than the electron drift speed it experiences a drag force.

The electron drift speed is related to the current density, $j$, by $v_{e}=j / n e$. Therefore, using the above measured value of the electron drag coefficient for aluminum, $B_{e}=1.4 \times 10^{-6}$ 
sPa, and a current density of $j=10^{10} \mathrm{~A} \mathrm{~m}^{-2}$, we obtain the current-induced force per unit length on a stationary dislocation $F_{e}=4.8 \times 10^{-7} \mathrm{~N} \mathrm{~m}^{-1}$. Equating this force per unit length to an equivalent shear stress acting on the dislocation, multiplied by its Burgers vector, we find it is equivalent to a shear stress of $1.7 \mathrm{kPa}$. Even if $B_{e}$ were two orders of magnitude larger, the equivalent stress would be only a little more than atmospheric pressure. These stresses are too small to make a sufficient difference to plastic deformation to explain the stress drops observed during electropulsing experiments.

In the remainder of this Sec. we will argue the following points. (1) The above reasoning that led to the estimate for the current-induced force per unit length on a stationary dislocation is flawed. (2) If there is a current-induced force on a dislocation it is not the "electron wind force." The current must generate a force dipole on a dislocation to make it glide. (3) If electromigration of dislocations exists it cannot explain the stress drops observed by electropulsing because it does not bring about a change of plastic strain.

1. The breakdown of the argument based on the equivalence of the crystal and dislocation rest frames

Equation $(\mathrm{C} 1)$ is based on changing rest frames between that of the crystal and a moving dislocation. It assumes the atomic structures of a static dislocation and a dislocation in motion are equivalent. They are equivalent only if a dislocation in motion is a rigidly displaced exact copy of a static dislocation. That is unlikely ever to be true. For example, dislocation motion may involve the creation and motion of kinks. Nonplanar static dislocation cores may have to transform into planar cores before they can glide. However, even if the core is planar and lying in the slip plane, as in aluminum and most other FCC metals, it is still not true. To see this consider a gliding edge dislocation with a planar core. Imagine a Maxwell demon sitting on an atom in the row terminating the extra half-plane. In the rest frame of the dislocation the demon swings backwards and forwards every time the dislocation advances by one atomic spacing along the slip plane. The swings are associated with breaking one bond and forming another with atoms on the other side of the slip plane. The amplitude of the swings decreases as the width of the core increases because more atoms undergo smaller movements to effect the relative displacement by the Burgers vector on either side of the slip plane. Since a gliding dislocation is very unlikely to be a rigid displacement of a static dislocation there is no justification for Eq. (C1). The above estimate of the current-induced force using the electron drag coefficient is therefore without foundation.

\section{The nature of current-induced forces on dislocations}

Our view of current-induced forces on a defect is based on the modern theory of electromigration [28-31]. To illustrate the modern theory consider an isolated point defect in a large metal crystal. We assume the crystal is in a fully relaxed configuration before a constant current is passed through the crystal. Current-carrying electrons are scattered by the point defect and atoms close to it. The scattering redistributes electronic charge around the point defect. In the steady state, the charge redistribution is self-consistent with the total potential acting on the electrons. The current-induced force on the point defect is then exactly equal to the charge of its nucleus multiplied by the self-consistent electric field at its nucleus.
For a scatterer in a free-electron gas, the current-induced force is identically equal to the rate of momentum transfer from the electrons. This force is often called the electron-wind force. For a point defect in a crystalline metal the picture is more complicated, because electrons are scattered not only by the point defect but also by surrounding atoms. For interacting electrons it has been shown that there are counter-balancing forces distributed on the surrounding atoms whose sum is equal and opposite to the force on the point defect itself [20]. As a result of these counter-balancing forces the current does not exert a net force on the metal in the steady state. This is discussed further in Sec. IV.

There is also a distribution of forces imparted to atoms in a dislocation by current-carrying electrons. By summing the forces on atoms in the dislocation core we obtain a currentinduced force on the dislocation. In a free-electron model, this would be the wind force. However, this force cannot make a dislocation glide because it is a force monopole by construction. It will only strain the region around the dislocation elastically. That is because the mechanism by which a dislocation glides involves shear on its slip plane. For a dislocation to be induced to glide, there must be a shear stress resolved on its slip plane in the direction of its Burgers vector. To generate a shear stress there must be current-induced forces with components parallel and antiparallel to the Burgers vector on either side of the slip plane, forming a dipole of forces. If such a current-induced shear stress is generated it can be substituted into the Peach-Koehler formula to calculate the current-induced force per unit length on the dislocation. The Peach-Koehler formula is as follows:

$$
\mathbf{f}_{P K}=(\sigma \mathbf{b}) \times \widehat{\xi},
$$

where the current-induced force per unit length acting on the dislocation is now identified with the Peach-Koehler force arising from the current-induced stress tensor, $\sigma$. The Burgers vector is $\mathbf{b}$ and $\widehat{\xi}$ is the direction of the dislocation line.

A resolved shear stress may be generated at an edge dislocation by a current, as illustrated in Fig. 11. At the terminating half-plane of the dislocation atoms are closer together than in the bulk, so the current flows more easily between them, creating an electrostatic dipole as shown in the figure. Beneath the terminating half plane atoms are further apart and the current flows less easily between them creating an electrostatic dipole in the opposite sense. The quadrupolar distribution of potential creates a dipole of forces across the slip plane, and hence a shear stress on atoms in the dislocation core. The current-induced shear stress in Fig. 11 drives the dislocation in the same direction as the electron current flow.

It is less clear that such a dipolar distribution of forces can be created at a screw dislocation, which suggests it will probably experience a smaller current-induced force than an edge dislocation.

3. Electromigration of dislocations does not increase plastic strain

Consider the edge dislocation depicted in Fig. 11. If we rotate the crystal by $180^{\circ}$ about the dashed line the extra half plane is below the slip plane and the signs of the dipoles above and below the slip plane are reversed. This reverses the stress $\sigma$ acting on the dislocation. The line direction $\widehat{\xi}$ of the dislocation is also reversed by this operation, but the Burgers 


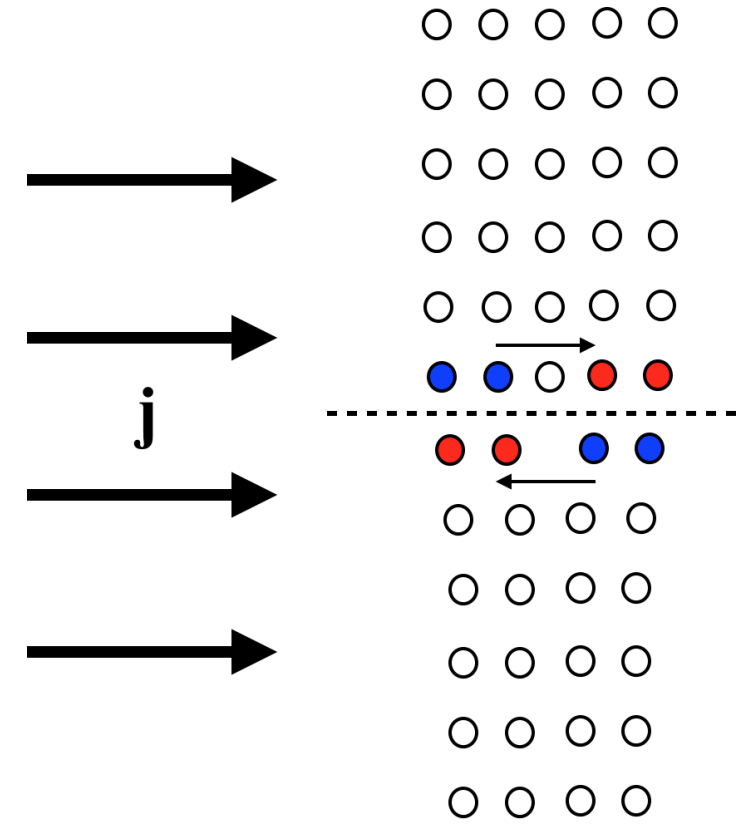

FIG. 11. Sketch to illustrate a current-induced electrostatic quadrupole at an edge dislocation. The large arrows show an incident electron current of density $\mathbf{j}$ from the left. The red (blue) atoms signify a higher (lower) electrostatic potential for electrons. The quadrupole generates forces, shown by small arrows, on atoms either side of the slip plane (broken line) in the dislocation core. These forces create a shear stress on the dislocation core. From Sutton [24].

vector $\mathbf{b}$ of the dislocation remains the same. Therefore the Peach-Koehler force, Eq. (C2), acting on the dislocation does not change after this rotation by $180^{\circ}$.

The Burgers vector $\mathbf{b}$ is reversed if the crystal is rotated by $180^{\circ}$ about the dislocation line. The current-induced shear stress $\sigma$ acting on the dislocation is reversed because the extra half plane is again beneath the slip plane. However, the direction $\widehat{\xi}$ of the dislocation line does not change. The result is that the current-induced Peach-Koehler force on the dislocation is again unchanged.

It follows that for an edge dislocation the same currentinduced force per unit length is induced regardless of the signs of its Burgers vector and line direction. If there are currentinduced forces on screw dislocations they are likely to display the same symmetries as shown above for edge dislocations. Therefore electromigration of equal numbers of positive and negative dislocations does not increase plastic strain because they move in the same direction under the influence of the current. Similarly dislocation loops will be translated by the current but not expanded. If electromigration of dislocations does not lead to increased plastic strain it cannot explain the larger stress drops observed in the plastic part of the stressstrain curve than those observed in the elastic part.

The approach we take in Sec. V is based on the PeachKoehler formula of Eq. (C2). However, the stress field $\sigma$ is not generated by the interaction between current-carrying electrons and dislocations but by Lorentz body forces acting on all atoms in the sample. Consequently dislocations of opposite signs move in opposite directions and dislocation loops expand when these stresses act upon them.

\section{APPENDIX D: JOULE HEATING}

Sceptics of the load drops observed during current pulsing often assert they are merely a result of Joule heating. They claim that the passage of such high current densities heats the metal. The heating leads to thermal expansion which reduces the tensile stress in a specimen subjected to a constant tensile strain. It may also facilitate thermally activated slip processes, leading to larger stress drops in the plastic part of the stress-strain curve. However, Troitskii [1] observed stress drops under compression loading when the compressive stress would be expected to rise if the current pulses produced only thermal expansion.

A particularly good example of the scepticism was provided by the experiments reported by Goldman et al. [44]. They carried out tensile tests on lead crystals at $4.2 \mathrm{~K}$, a temperature at which lead is a superconductor. They monitored the voltage across the specimen as current pulses were passed through it with densities between $2 \times 10^{6}$ and $8 \times 10^{6} \mathrm{~A} \mathrm{~m}^{-2}$. At the same time, they monitored the load in the specimen with a high sensitivity equal to $0.01 \%-0.03 \%$ of the yield stress. At the lower current densities the specimen remained superconducting and it displayed no load drops during the current pulses. At the higher current densities. the specimen became normal and then it did display load drops. The authors argued that when the specimen became normal the current pulses heated the specimen, because then it had a resistance. However, as long as the specimen remained superconducting there was no heating, because it had no resistance. They concluded that the load drops were associated with Joule heating. They found the specimen remained superconducting at higher current densities after further plastic deformation. Again they found no load drops at a higher current density where the specimen remained superconducting. However, at an even higher current density, the specimen became normal and load drops were observed. They argued this shows the load drops are dependent only on whether the specimen is in a normal or superconducting state, and not on a critical current density.

The current densities used in the experiments of Goldman et al. [44] are orders of magnitude less than those used by Troitskii and by Conrad and coworkers. The latter reported critical current densities for electroplasticity that were much greater than those used by Goldman et al. It is quite possible, therefore, that no current-facilitated plasticity occurred in the experiments of Goldman et al. and that the load drops were indeed entirely a result of thermal expansion in the normal state.

\section{APPENDIX E: THE STATIC PINCH EFFECT}

Under the influence of a constant current along a cylindrical wire, there is a radial contraction due to the static pinch effect. If the wire is under tension at constant length the tensile stress in the wire is reduced by the pinch effect. That is because if the wire were not constrained to be of constant length the radial compression would increase its length through the Poisson effect. However, since the wire is constrained to have a constant length the Poisson effect results in a compressive stress along the wire, which reduces the applied tensile stress. 
Therefore it is conceivable that the static pinch effect can account for the stress drops observed during current-pulsing in the elastic part of the stress-strain curve, which may also account for part of the current-induced stress drops observed when the wire is deformed plastically.

Consider a long straight cylindrical wire of radius $r_{m}$. Introduce a cylindrical coordinate system $(r, \phi, z)$ with the $z$-axis along the axis of the wire. We assume the wire is homogeneous and elastically isotropic. The wire is carrying a constant current of density $j_{0}$, which is uniformly distributed in the wire. The magnetic field inside the wire at a radius $r$ is

$$
\mathbf{B}=\frac{\mu j_{0} r}{2} \widehat{\boldsymbol{\phi}},
$$

where $\widehat{\phi}$ is a unit vector along the azimuthal direction. The Lorentz force on the conduction electrons per unit volume, $\mathbf{f}_{L}=\mathbf{j} \times \mathbf{B}$, is then

$$
\mathbf{f}_{L}=-\frac{\mu j_{0}^{2} r}{2} \hat{\mathbf{r}}
$$

In Sec. IV B, we showed that the Lorentz force on the conduction electrons is transferred to the ionic cores of the metal creating a body force on them. This radial body force generates radial displacements $u(r)$. The equation of mechanical equilibrium, in cylindrical coordinates and with radial symmetry, is as follows:

$$
\frac{d \sigma_{r r}}{d r}+\frac{\sigma_{r r}-\sigma_{\phi \phi}}{r}=-f_{L} .
$$

The following strains are present: $e_{r r}=u^{\prime}(r)$ and $e_{\phi \phi}=$ $u(r) / r$ where $u(r)$ is the radial elastic displacement and the prime denotes differentiation. In experiments where the length of the wire is increased at a fixed rate its length is not allowed to change in response to stresses created within it. The translational invariance along $z$ then ensures plane strain, so that $e_{z z}=0$. In isotropic elasticity the stresses are related to the strains by Hooke's law:

$$
\begin{aligned}
\sigma_{r r} & =2 G_{m} e_{r r}+\lambda\left(e_{r r}+e_{\phi \phi}\right), \\
\sigma_{\phi \phi} & =2 G_{m} e_{\phi \phi}+\lambda\left(e_{r r}+e_{\phi \phi}\right), \\
\sigma_{z z} & =v\left(\sigma_{r r}+\sigma_{\phi \phi}\right),
\end{aligned}
$$

where $G_{m}$ is the shear modulus, $\lambda=2 G_{m} v /(1-2 v)$ and $v$ is Poisson's ratio. The expression for $\sigma_{z z}$ follows from the assumption of plane strain. Substituting these relations between the stresses and strains and between the strains and the displacement field $u(r)$ into the equilibrium equation, Eq. (E3), we obtain the following differential equation for the displacement field:

$$
u^{\prime \prime}+\frac{u^{\prime}}{r}-\frac{u}{r^{2}}=\frac{(1-2 v)}{4(1-v)} \frac{\mu j_{0}^{2}}{G_{m}} r .
$$

The solution is as follows:

$$
u=C_{1} r+\frac{(1-2 v)}{32(1-v)} \frac{\mu j_{0}^{2}}{G_{m}} r^{3},
$$

where $C_{1}$ is an arbitrary constant to be determined by the boundary condition that $\sigma_{r r}\left(r_{m}\right)=0$. The general solution of Eq. (E5) includes a term $C_{2} / r$, where $C_{2}$ is an arbitrary constant. We equate $C_{2}$ to zero because there is no singularity at $r=0$.

Equating $\sigma_{r r}$ to zero at $r=r_{m}$ yields the following equation for $C_{1}$ :

$$
C_{1}=-\frac{(3-2 v)(1-2 v)}{32(1-v)} \frac{\mu j_{0}^{2}}{G_{m}} r_{m}^{2}
$$

Therefore the stresses $\sigma_{r r}(r)$ and $\sigma_{\phi \phi}(r)$ are as follows:

$$
\begin{gathered}
\sigma_{r r}(r)=-\frac{(3-2 v)}{16(1-v)}\left(r_{m}^{2}-r^{2}\right) \mu j_{0}^{2}, \\
\sigma_{\phi \phi}(r)=-\frac{\left((3-2 v) r_{m}^{2}-(1+2 v) r^{2}\right)}{16(1-v)} \mu j_{0}^{2} .
\end{gathered}
$$

Using Eq. (E4), we obtain

$$
\sigma_{z z}(r)=-\frac{v}{8(1-v)}\left((3-2 v) r_{m}^{2}-2 r^{2}\right) \mu j_{0}^{2} .
$$

Since $v \leqslant \frac{1}{2}$ we see that $\sigma_{z z}(r)$ is compressive at all radii. The mean value of $\sigma_{z z}(r)$ is

$$
\left\langle\sigma_{z z}\right\rangle=-v \mu j_{0}^{2} r_{m}^{2} / 4
$$

Assuming $v=1 / 3, \mu=4 \pi \times 10^{-7} \mathrm{~N} \mathrm{~A}^{-2}, j_{0}=3 \times 10^{9}$ $\mathrm{A} \mathrm{m}^{-2}$, and $r_{m}=5 \times 10^{-4} \mathrm{~m}$, we obtain $\left\langle\sigma_{z z}\right\rangle \approx 0.2 \mathrm{MPa}$, and the maximum value of $\sigma_{z z}$ is approximately $0.4 \mathrm{MPa}$. These stresses are too small by more than an order of magnitude to account for the stress drops observed in the elastic part of the stress-strain curve by Okazaki et al. [12].

In the experiments of Troitskii [1] and Okazaki et al. [10] the nominal strain rates imposed by the Instron machine were $1.1 \times 10^{-4}$ and $1.7 \times 10^{-4} \mathrm{~s}^{-1}$ respectively. For a pulse of duration $10^{-4} \mathrm{~s}$ the strain increment was therefore between $1.1 \times 10^{-8}$ and $1.7 \times 10^{-8}$. If the Young's modulus of the wire is of order $100 \mathrm{GPa}$, the strains produced in the static pinch effect by stresses of order $0.1 \mathrm{MPa}$ are of order $10^{-6}$, which is two orders of magnitude larger than the strain increment imposed by the Instron machine during the pulse. It follows that even for the small stresses of the static pinch effect the wire may be treated as having a constant length throughout a pulse of $10^{-4} \mathrm{~s}$ duration. As we saw, in Sec. V D, the stresses created in the dynamic pinch effect can be much larger than those in the static pinch effect. The argument for treating the wires as having a constant length throughout the pulse is then even stronger.

Equating the force $-\rho_{0} \mathcal{E}(r)$ on conduction electrons due to the Hall field to $-f_{L}=\mu j_{0}^{2} r / 2$ (see Eq. (E2)) we obtain $\mathcal{E}(r)=-\mu j_{0}^{2} r /\left(2 \rho_{0}\right)$. Taking the divergence of the radial electric field $\mathcal{E}(r)$ we find it is generated by an extremely small uniform negative charge density $\delta \rho_{0}$ throughout the wire given by $\delta \rho_{0} / \rho_{0}=-\mu_{r} v_{d}^{2} / c^{2}$. This extremely small uniform charge density inside the metal can be neutralized by a small surface charge density, $\mu_{r} \rho_{0} r v_{d}^{2} /\left(2 c^{2}\right)$, which does not affect the electric field inside the wire. 
[1] O. A. Troitskii, Electromechanical effect in metals, Zh. Eksp. Teor. Fiz. Pisma Redaktsiiu 10, 18 (1969).

[2] A. F. Sprecher, S. L. Mannan, and H. Conrad, Overview no. 49: On the mechanisms for the electroplastic effect in metals, Acta Metall. 34, 1145 (1986).

[3] H. Conrad, Electroplasticity in metals and ceramics, Mater. Sci. Eng.: A 287, 276 (2000).

[4] S. D. Antolovich and H. Conrad, The effects of electric currents and fields on deformation in metals, ceramics, and ionic materials: An interpretive survey, Mater. Manuf. Processes 19, 587 (2004).

[5] N. K. Dimitrov, Y. Liu, and M. F. Horstemeyer, Electroplasticity: A review of mechanisms in electro-mechanical coupling of ductile metals, Mech. Adv. Mater. Struct. (2020), doi:10.1080/15376494.2020.1789925.

[6] S. Zhao, R. Zhang, Y. Chong, X. Li, A. Abu-Odeh, E. Rothchild, D. C. Chrzan, M. Asta, J. W. Morris, and A. M. Minor, Defect reconfiguration in a $\mathrm{Ti}-\mathrm{Al}$ alloy via electroplasticity, Nat. Mater. 20, 468 (2021).

[7] A. A. Shibkov, A. A. Denisov, M. A. Zheltov, A. E. Zolotov, and M. F. Gasanov, The electric current-induced suppression of the Portevin-Le Chatelier effect in Al-Mg alloys, Mater. Sci. Eng.: A 610, 338 (2014).

[8] L. Guan, G. Tang, and P. K. Chu, Recent advances and challenges in electroplastic manufacturing processing of metals, J. Mater. Res. 25, 1215 (2010).

[9] H.-D. Nguyen-Tran, H.-S. Oh, S.-T. Hong, H. N. Han, J. Cao, S.-H. Ahn, and D.-M. Chun, A review of electrically-assisted manufacturing, Int. J. Precis. Eng. Manuf.-Green Technol. 2, 365 (2015).

[10] K. Okazaki, M. Kagawa, and H. Conrad, A study of the electroplastic effect in metals, Scr. Metall. 12, 1063 (1978).

[11] V. E. Gromov, L. B. Zuev, V. I. Bazaikin, and V. Ya. Tsellermaer, Laws of electrical stimulation of plastic deformation of metals and alloys at various structural levels, Russ. Phys. J. 39, 237 (1996).

[12] K. Okazaki, M. Kagawa, and H. Conrad, Additional results on the electroplastic effect in metals, Scr. Metall. 13, 277 (1979).

[13] M. Molotskii and V. Fleurov, Magnetic effects in electroplasticity of metals, Phys. Rev. B 52, 15829 (1995).

[14] K. Okazaki, M. Kagawa, and H. Conrad, Effects of strain rate, temperature and interstitial content on the electroplastic effect in titanium, Scr. Metall. 13, 473 (1979).

[15] N. E. Kir'yanchev, O. A. Troitskii, and S. A. Klevtsur, Electroplastic deformation of metals, Strength Mater. 15, 709 (1983).

[16] O. A. Troitskii and V. I. Spitsyn, Investigation of the electroplastic deformation of a metal by the method of stress relaxation and creep, Sov. Phys. Doklady 21, 111 (1976).

[17] M. I. Molotskii, R. E. Kris, and V. Fleurov, Internal friction of dislocations in a magnetic field, Phys. Rev. B 51, 12531 (1995).

[18] M. Molotskii and V. Fleurov, Spin Effects in Plasticity, Phys. Rev. Lett. 78, 2779 (1997).

[19] M. I. Molotskii, Theoretical basis for electro- and magnetoplasticity, Mater. Sci. Eng.: A 287, 248 (2000).

[20] J. Hoekstra, A. P. Sutton, and T. N. Todorov, Counterbalancing forces in electromigration, J. Phys.: Condens. Matter 14, L137 (2002).
[21] W. R. Smythe, Static and Synamic Electricity (McGraw-Hill, New York, 1950).

[22] W. F. Gale and T. C. Totemeier, Smithells Metals Reference Book, 8th ed. (Elsevier, Oxford, 2004).

[23] F. Fiorillo, Measurement and Characterization of Magnetic Materials (Elsevier, Amsterdam, 2004).

[24] A. P. Sutton, Physics of Elasticity and Crystal Defects (Oxford University Press, New York, 2020).

[25] V. Psyk, D. Risch, B. L. Kinsey, A. E. Tekkaya, and M. Kleiner, Electromagnetic forming - a review, J. Mater. Process. Technol. 211, 787 (2011).

[26] P. L. Kapitza, A method of producing strong magnetic fields, Proc. R. Soc. A 105, 691 (1924).

[27] O. A. Troitskii, O. B. Skvortsov, and V. I. Stashenko, Measurements of vibrations produced by current pulses in elements of electrical equipment, Russ. Electr. Eng. 89, 143 (2018).

[28] R. Landauer and J. W. Woo, Driving force in electromigration, Phys. Rev. B 10, 1266 (1974).

[29] R. Landauer, Residual resistivity dipoles, Z. Phys. B 21, 247 (1975).

[30] R. S. Sorbello, Theory of electromigration, Solid State Phys. 51, 159 (1997).

[31] T. N. Todorov, Tight-binding simulation of current-carrying nanostructures, J. Phys.: Condens. Matter 14, 3049 (2002).

[32] I. L. Bataronov and A. M. Roshchupkin, Electroplastic deformation of metals and dynamic pinch effect, Steel Transl. 23, 45 (1993).

[33] A. M. Roshchupkin and I. L. Bataronov, Physical basis of the electroplastic deformation of metals, Russ. Phys. J. 39, 230 (1996).

[34] U. E. Steiner and T. Ulrich, Magnetic field effects in chemical kinetics and related phenomena, Chem. Rev. 89, 51 (1989).

[35] A. Valladares and A. P. Sutton, First principles simulations of antiphase defects on the SP $90^{\circ}$ partial dislocation in silicon, J. Phys.: Condens. Matter 18, 3735 (2006).

[36] O. Y. Kontsevoi, Y. N. Gornostyrev, O. N. Mryasov, A. J. Freeman, M. I. Katsnelson, and A. V. Trefilov, Electron localization on dislocations in metals: Real-space first-principles calculations, Phys. Rev. B 64, 134103 (2001).

[37] P. W. Anderson, Localized magnetic states in metals, Phys. Rev. 124, 41 (1961).

[38] H. Kojima and T. Suzuki, Electron Drag and Flow Stress in Niobium and Lead at 4.2K, Phys. Rev. Lett. 21, 896 (1968).

[39] J. M. Galligan, How to distinguish dislocation drag processes from thermal activation, Phys. Status Solidi (b) 172, 19 (1972).

[40] A. Hikata, R. A. Johnson, and C. Elbaum, Interaction of dislocations with electrons and with phonons, Phys. Rev. B 2, 4856 (1970).

[41] V. Y. Kravchenko, Effect of directed electron beam on moving dislocations, Sov. Phys. JETP 24, 1135 (1967).

[42] K. M. Klimov, G. D. Shnyrev, and I. I. Novikov, "Electroplasticity" of metals, Sov. Phys. Doklady 19, 787 (1975).

[43] A. M. Roshchupkin, V. E. Miloshenko, and V. E. Kalinin, Effect of electrons on the motion of dislocations in metals, Sov. J. Exp. Theor. Phys. Lett. 29, 435 (1979).

[44] P. D. Goldman, L. R. Motowidlo, and J. M. Galligan, The absence of an electroplastic effect in lead at $4.2 \mathrm{~K}$, Scr. Metall. 15, 353 (1981). 